\title{
Pacific
}

Journal of

Mathematics

\section{THE COHOMOLOGY OF HIGHER-DIMENSIONAL SHIFTS OF} FINITE TYPE

KLAUS SCHMIDT

Volume 170 No. 1 


\title{
THE COHOMOLOGY OF HIGHER-DIMENSIONAL SHIFTS OF FINITE TYPE
}

\author{
KLAUS SCHMIDT
}

\begin{abstract}
We discuss the cohomology of higher-dimensional shifts of finite type, and prove the following: if a $d$-dimensional shift of finite type $X$ has a rich supply of homoclinic points and a certain specification property, then every Hölder cocycle for shift-action of $\mathbb{Z}^{d}$ on $X$ with values in a locally compact, second countable group with a doubly invariant metric is Höldercohomologous to a homomorphism. The result is illustrated with a number of examples.
\end{abstract}

\section{Introduction.}

One of many surprising differences between $\mathbb{Z}$-actions and $\mathbb{Z}^{d}$-actions for $d>1$ is that certain first cohomology groups of the latter actions may be very small. The first example which came to my attention was due to J.W. Kammeyer ([Kam1], [Kam2]), who proved that every continuous cocycle for the shift-action of $\mathbb{Z}^{d}$ on the full $d$-dimensional $k$-shift with values in $\mathbb{Z} / 2 \mathbb{Z}$ is continuously cohomologous to a homomorphism from $\mathbb{Z}^{d}$ to $\mathbb{Z} / 2 \mathbb{Z}$ (the terminology is explained in Section 3 ). A second instance of this phenomenon appeared in a recent paper by A. Katok and R.J. Spatzier [KaSp]: every real-valued Hölder cocycle for an Anosov action of $\mathbb{Z}^{d}$ on a compact manifold is Hölder-cohomologous to a homomorphism, in complete contrast to the rich supply of nontrivial Hölder cocycles for a single Anosov map. Based on ideas in that paper and the machinery developed in [Sch1], [KS1], and [KS3], it was proved in [KaS] that every real-valued Hölder cocycle (or, more generally, every real-valued cocycle with summable variation) for an expansive and (topologically) mixing $\mathbb{Z}^{d}$-action by automorphisms of a compact, abelian group is Hölder- (or continuously) cohomologous to a homomorphism. Since every expansive $\mathbb{Z}^{d}$-action by automorphisms of a compact, zero-dimensional, abelian group is topologically conjugate to the shift-action of $\mathbb{Z}^{d}$ on a shift of finite type, the paper [KaS] provides further examples of shifts of finite type with small cohomology groups. The situation is different if one considers continuous cocycles with values in a finite, abelian group: in Example 5.3 below we show that expansive and mixing $\mathbb{Z}^{d}$-actions by automorphisms of a compact, zero-dimensional, abelian group may have 
Hölder (i.e. continuous) cocycles with values in a finite group which are not cohomologous to homomorphisms.

The purpose of this paper is to present conditions on a $d$ dimensional shift of finite type $X$, under which ever Hölder cocycle (or every cocycle with summable variation) for the shift-action $\sigma$ of $\mathbb{Z}^{d}$ on $X$ with values in a locally compact group with a given doubly invariant metric is trivial, i.e. cohomologous to a homomorphism (the existence of a double invariant metric is required to make sense of the notion of a Hölder cocycle; if $G$ is discrete, then every continuous cocycle with values in $G$ is automatically Hölder). The conditions are that there exists a point $x \in X$ whose set of homoclinic points is dense in $X$ and has a certain specification property (a point $y \in X$ is homoclinic to $x$ if $\lim _{\mathbf{m} \rightarrow \infty} \delta\left(T_{\mathbf{m}}(x), T_{\mathbf{m}}(y)\right)=0$ for some metric $\delta$ on $X)$. Although these conditions force the shift to have positive entropy, one can conclude from this result that certain zero entropy shifts of finite type must have trivial Hölder cohomology (cf. Section 5).

This paper is organised as follows: In Section 2 we define the Gibbs (or homoclinic) equivalence relation $\Delta_{X}$ on a $d$-dimensional shift of finite type $X$, discuss its connection with entropy and periodic points, and introduce the notion of $\mathbf{n}$-specification of $\Delta_{X}$. Section 3 contains the main results (Theorem 3.2 and Corollaries 3.3-3.4) about triviality of cocycles for an expansive $\mathbb{Z}^{d}$-action $T$ on a compact, zero-dimensional space $X$ under the assumption that the equivalence relation $\Delta_{X}$ is topologically transitive and has $\mathbf{n}$-specification for some nonzero element $\mathbf{n} \in \mathbb{Z}^{d}$. From the proof of Proposition 3.1 (which forms the main tool for the proof of Theorem 3.2) it appears that there is a close connection between the triviality of the fundamental group of the shift in the sense of [GePr] and the triviality of all Hölder cocycles (Corollary 3.6), but the precise extent of this connection is not at all clear. In Section 4 we discuss a variety of classical higher-dimensional shifts of finite type, determine for each of them whether it satisfies the conditions of Corollary 3.3, and find nontrivial cocycles in all cases where the conditions are not satisfied. The specific examples are the full shifts ([Kam1], [Kam2]), tilings of $\mathbb{Z}^{2}$ by dominoes (or dimers), the colourings of infinite chessboards or lattices with at least 3 colours, the 'square ice' model and other related shifts of finite type, such as lozenge tilings, and the higherdimensional golden mean shifts. The chessboard colourings show that the triviality of all Hölder cocycles is quite a subtle phenomenon: if one uses 3 colours, then there exist nontrivial Hölder cocycles with values in every locally compact, second countable, abelian group; however, if $n \geq 4$, every Hölder cocycle with values in any locally compact group must be trivial. Furthermore, all these shifts have finite-to-one factors with nontrivial Hölder cohomology. In particular, the 'square ice' model is a three-to-one factor of 
the set of chessboard colourings with three colours and admits nontrivial integer-valued Hölder cocycles, and one can obtain nontrivial cocycles on the set of lozenge tilings by embedding it as a subshift of the 'square ice' model, and by restricting the cocycles on that shift space to the lozenge tilings. In Section 5 we turn to shifts of finite type with zero entropy and investigate their cohomology. Example 5.1 is due to W. Parry and shows that a two-dimensional shift of finite type with zero entropy may have nontrivial real (or integer) valued Hölder cocycles. In Examples 5.3 (1)-(7) we present some shifts of finite type with zero topological entropy introduced in [Led], [KS2], and [KS3], for which every real (or integer) valued cocycle with summable variation is trivial ([KaS $]$ ), but which may have nontrivial continuous (and hence Hölder) cocycles with values in finite, abelian groups. These examples are based on an algebraic criterion for the existence of certain nontrivial cocycles for such actions (Proposition 5.2). Other examples in this section are a zero entropy shift of finite type for which every Hölder cocycle with values in a locally compact group is trivial (Example 5.4), and a shift which has nontrivial Hölder cocycles with values in certain nonabelian groups, but for which every such cocycle with values in a discrete abelian group is trivial (Proposition 5.5).

There are a number of open questions attached to these examples. Every example in Section 4 with trivial Hölder cohomology also has trivial fundamental group (cf. [GePr]). Where the cohomology is nontrivial it seems reasonable to conjecture some link between the fundamental group of the shift $X$ and those groups $G$ for which there exists a nontrivial cocycle $c$ on $X$ with values in $G$; however, this link is likely to be quite complicated. The domino tilings provide an example where the fundamental group is abelian (and isomorphic to $\mathbb{Z}$ ), but there does not appear to exist a nontrivial Hölder cocycle $c$ on the set of all such tilings with values in an abelian group; there are, however, nontrivial cocycles with values in some nonabelian groups. Example 5.4 is a three-dimensional shift of finite type with trivial cohomology, but with nontrivial fundamental group. Finally, even if there exist nontrivial cocycles on some of these shift spaces, then the first cohomology of the shift-actions appears to be very small; at this stage there are, however, no precise results in this direction.

The triviality of all Hölder cocycles (or of all cocycles with summable variation) for a shift of finite type $X$ has obvious dynamical implications for suspensions of that system, as well as for certain factor maps. For example, if the shift-action $\sigma$ of $\mathbb{Z}^{d}$ on $X$ is individually mixing (i.e. if $\sigma_{\mathbf{n}}$ is topologically mixing for every nonzero $\mathbf{n} \in \mathbb{Z}^{d}$ ), and if every continuous cocycle for $\sigma$ with values in a finite group is trivial, then $\sigma$ has no continuous, individually mixing $G$-extensions for any discrete group $G$ (Corollary 3.4), and there 
cannot exist an (individually) mixing shift of finite type $Y$ and a continuous, shift-commuting, open $\phi: Y \longmapsto X$ which is everywhere $n$-to-one for some $n \geq 2$ (Corollary 3.5).

On a more philosophical level, the triviality of all Hölder cocycles can be interpreted as a 'rigidity'-property. This point of view is taken in [KaSp], but is also implicit in the link between cocycles and height functions on spaces of tilings (such as the domino and lozenge tilings, or the square ice model) discussed in [Thu]: if a cocycle is trivial, the Lipschitz surface defined by its height function is essentially flat, and in the absence of nontrivial cocycles there are no interesting 'deformations' of these surfaces. Rigidity assertions of this nature are quite natural in the context of the algebraic $\mathbb{Z}^{d}$-actions investigated in [KaSp] or [KaS], but it strikes me as surprising to find analogous results for certain higher-dimensional shifts of finite type, which seem far removed from any algebraic setting.

\section{Subshifts and equivalence relations.}

Let $X$ be a compact, metrizable space. A Borel equivalence relation $\mathbf{R} \subset$ $X \times X$ is an equivalence relation which is a Borel set ([FeM]). Since we shall never deal with equivalence relations which are not Borel we use the term equivalence relation to denote a Borel equivalence relation. If $\mathbf{R} \subset X \times X$ is an equivalence relation we write $\mathbf{R}(x)=\left\{x^{\prime} \in X:\left(x, x^{\prime}\right) \in \mathbf{R}\right\}$ for the equivalence class of a point $x \in X$, and call $\mathbf{R}$ topologically transitive if $\mathbf{R}(x)$ is dense in $X$ for some $x \in X$, minimal if $\mathbf{R}(x)$ is dense in $X$ for every $x \in X$, trivial if $\mathbf{R}(x)=\{x\}$ for all $x \in X$, and nontrivial otherwise.

We are going to be interested in certain equivalence relations associated with higher-dimensional shifts of finite type as defined in [Rue] or [Sch2], for example. Let $d \geq 1, A$ a finite set (the alphabet), and let $A^{\mathbb{Z}^{d}}$ be the set of all maps $x: \mathbb{Z}^{d} \longmapsto A$. For every subset $F \subset \mathbb{Z}^{d}$ we denote by $\pi_{F}: A^{\mathbb{Z}^{d}} \longmapsto A^{F}$ the projection map which restricts each $x \in A^{\mathbb{Z}^{d}}$ to the set $F$. The space $A^{\mathbb{Z}^{d}}$ is compact in the product topology. We write a typical point $x \in A^{\mathbb{Z}^{d}}$ as $x=\left(x_{\mathbf{m}}\right)=\left(x_{\mathbf{m}}, \mathbf{m} \in \mathbb{Z}^{d}\right)$, where $x_{\mathbf{m}} \in A$ denotes the value of $x$ at $\mathbf{m}$, and define, for every $\mathbf{n} \in \mathbb{Z}^{d}$, a homeomorphism $\sigma_{\mathbf{n}}$ of $A^{\mathbb{Z}^{d}}$ by

$$
\left(\sigma_{\mathbf{n}}(x)\right)_{\mathbf{m}}=x_{\mathbf{m}+\mathbf{n}}
$$

for every $x=\left(x_{\mathbf{m}}\right) \in A^{\mathbb{Z}^{d}}$. The map $\sigma: \mathbf{n} \longmapsto \sigma_{\mathbf{n}}$ is the shift-action of $\mathbb{Z}^{d}$ on $A^{\mathbb{Z}^{d}}$, and a subset $X \subset A^{\mathbb{Z}^{d}}$ is shift-invariant if $\sigma_{\mathbf{n}}(X)=X$ for all $\mathbf{n} \in \mathbb{Z}^{d}$. A closed, shift-invariant set $X \subset A^{\mathbb{Z}^{d}}$ is a subshift, and $X$ is a shift of finite type (sft) if there exists a finite set $F \subset \mathbb{Z}^{d}$ such that

$$
X=\left\{x \in A^{\mathbb{Z}^{d}}: \pi_{F} \cdot \sigma_{\mathbf{n}}(x) \in \pi_{F}(X) \text { for every } \mathbf{n} \in \mathbb{Z}^{d}\right\} .
$$


If $X \subset A^{\mathbb{Z}^{d}}$ is a sft we may change the alphabet $A$, if necessary, and assume that

$$
F=I^{d}=\{0,1\}^{d} \subset \mathbb{Z}^{d} .
$$

The restriction of the shift-action $\sigma$ on $\mathbb{Z}^{d}$ on $A^{\mathbb{Z}^{d}}$ to a subshift $X \subset A^{\mathbb{Z}^{d}}$ will again be denoted by $\sigma$, or by $\sigma^{X}$ if there is any danger of confusion. A point $x \in X$ is periodic if the group $\left\{\mathbf{n} \in \mathbb{Z}^{d}: \sigma_{\mathbf{n}}(x)=x\right\}$ has finite index in $\mathbb{Z}^{d}$, and the subshift $X$ has dense periodic points $(d p p)$ if its set of periodic points is dense. A sft $X$ may not contain any periodic points, and this potential absence of periodic points is associated with some of the difficulties one encounters when dealing with $s f t$ 's in higher dimensions.

For the remainder of this section we fix an alphabet $A$ and a sft $X \subset A^{\mathbb{Z}^{d}}$, and consider the Gibbs (or homoclinic) equivalence relation $\Delta_{X} \subset X \times X$ defined by

$$
\Delta_{X}=\left\{\left(x, x^{\prime}\right) \in X \times X: x_{\mathbf{m}} \neq x_{\mathbf{m}}^{\prime} \text { for only finitely many } \mathbf{m} \in \mathbb{Z}^{d}\right\},
$$

which is connected with the topological entropy of the shift-action $\sigma$ of $\mathbb{Z}^{d}$ on $X$ defined by (2.1).

Proposition 2.1. If the topological entropy $h(\sigma)$ of the shift-action $\sigma$ of $\mathbb{Z}^{d}$ on $X$ is positive, then the Gibbs relation $\Delta_{X}$ is nontrivial. Furthermore, if $X$ has $\operatorname{dpp}$ and $h(\sigma)=0$, then $\Delta_{X}$ is trivial, and the restriction of $\sigma$ to any subgroup $\Gamma \simeq \mathbb{Z}^{d-1}$ in $\mathbb{Z}^{d}$ has finite topological entropy.

Proof. We assume without loss in generality that $X$ satisfies (2.2)-(2.3). For every $N \geq 0$ we set $Q(N)=\{-N, \ldots, N\}^{d} \subset \mathbb{Z}^{d}$ and write $\partial Q(N)=$ $Q(N) \backslash Q(N-1)$ for the boundary of $Q(N), N \geq 1$. If $|S|$ is the cardinality of a set $S$, then

$$
h(\sigma)=\lim _{N \rightarrow \infty} \frac{1}{|Q(N)|} \log \left|\pi_{Q(N)}(X)\right|
$$

(c.f. e.g. [LSW]). Suppose that $\Delta_{X}$ is trivial. Then $\pi_{Q(N)}(x)=\pi_{Q(N)}\left(x^{\prime}\right)$ whenever $\pi_{\partial Q(N)}(x)=\pi_{\partial Q(N)}\left(x^{\prime}\right)$, so that

$$
\left|\pi_{Q(N)}(X)\right|=\left|\pi_{\partial Q(N)}(X)\right| \leq|A|^{|\partial Q(N)|}
$$

for every $N \geq 1$. Since $|\partial Q(N)| /|Q(N)| \rightarrow 0$ as $N \rightarrow \infty$ we conclude that $h(\sigma)=0$.

Now suppose that $X$ has $d p p$, and that $\Delta_{X}$ is nontrivial. Choose an integer $N \geq 1$ for which there exist two distinct points $x, x^{\prime} \in X$ such that $x_{n}=x_{\mathbf{n}}^{\prime}$ for all $\mathbf{n} \in \mathbb{Z}^{d} \backslash Q(N-1)$, and choose a periodic point $y \in X$ and 
an integer $L>2 N$ such that $\pi_{Q(N)}(y)=\pi_{Q(N)}(x)$ and $\sigma_{L \mathbf{m}}(y)=y$ for every $\mathbf{m} \in \mathbb{Z}^{d}$. Then there exists, for every $z \in\{0,1\}^{\mathbb{Z}^{d}}$, a unique point $x(z) \in X$ with the following properties:

$$
\begin{aligned}
\pi_{Q(N)}\left(\sigma_{L \mathbf{m}}(x(z))\right) & =\pi_{Q(N)}(x) \text { for every } \mathbf{m} \in \mathbb{Z}^{d} \text { with } z_{\mathbf{m}}=0 \\
\pi_{Q(N)}\left(\sigma_{L \mathbf{m}}(x(z))\right) & =\pi_{Q(N)}\left(x^{\prime}\right) \text { for every } \mathbf{m} \in \mathbb{Z}^{d} \text { with } z_{\mathbf{m}}=1, \\
x(z)_{\mathbf{n}} & =y_{\mathbf{n}} \text { for every } \mathbf{n} \in \mathbb{Z}^{d} \backslash \bigcup_{\mathbf{m} \in \mathbb{Z}^{d}}(Q(N)+L \mathbf{m}) .
\end{aligned}
$$

In particular, $h(\sigma)>0$.

In order to prove the last assertion we assume for simplicity that $\Gamma=$ $\left\{\mathbf{n}=\left(n_{1}, \ldots, n_{d}\right) \in \mathbb{Z}^{d}: n_{d}=0\right\}$, write $\tau: \mathbf{n} \longmapsto \sigma_{\mathbf{n}}, \mathbf{n} \in \Gamma$, for the restriction of $\sigma$ to $\Gamma$, and set $e^{(d)}=(0, \ldots, 0,1)$. For every $M, N \geq 0$ we put $E(M)=\left\{k \mathbf{e}^{(d)}:|k| \leq M\right\}, Q(N)^{\prime}=Q(N) \cap \Gamma, R(M, N)=Q(N)^{\prime}+E(M)$, $\partial R(M, N)=R(M, N) \backslash R(M-1, N-1), M, N \geq 1$, and write $\mathcal{P}_{M}=$ $\left\{B_{s}=\pi_{E(M)}^{-1}(s): s \in \pi_{E(M)}(X)\right\}$ for the partition of $X$ defined by the coordinates in $E(M)$. Since $\pi_{R(M, N)}(x)=\pi_{R(M, N)}\left(x^{\prime}\right)$ whenever $\pi_{\partial R(M, N)}(x)=$ $\pi_{\partial R(M, N)}\left(x^{\prime}\right)$ we have, in the usual notation, that

$$
\begin{aligned}
h(\tau) & =\sup _{M \geq 1} \lim _{N \geq 1} \frac{1}{\left|Q(N)^{\prime}\right|} \log \left|\bigvee_{\mathbf{n} \in Q(N)^{\prime}} \sigma_{\mathbf{n}}\left(\mathcal{P}_{M}\right)\right| \\
& =\sup _{M \geq 1} \lim _{N \geq 1} \frac{1}{\left|Q(N)^{\prime}\right|} \log \left|\pi_{R(M, N)}(X)\right| \\
& =\sup _{M \geq 1} \lim _{N \geq 1} \frac{1}{\left|Q(N)^{\prime}\right|} \log \left|\pi_{R(M, N)}(X)\right| \leq 2 \log |A|<\infty .
\end{aligned}
$$

The proof for an arbitrary subgroup $\Gamma \simeq \mathbb{Z}^{d-1}$ in $\mathbb{Z}^{d}$ is completely analogous, but notationally more complicated.

We write $\|\cdot\|$ and $\langle\cdot, \cdot\rangle$ for the Euclidean norm and inner product on $\mathbb{R}^{d} \supset$ $\mathbb{Z}^{d}$, put $\mathbf{0}=(0, \ldots, 0) \in \mathbb{Z}^{d}$, and set $\mathbf{B}(r)=\left\{\mathbf{n} \in \mathbb{Z}^{d}:\|\mathbf{n}\| \leq r\right\}, r \geq 0$. If $B, B^{\prime} \subset \mathbb{Z}^{d}$ are nonempty sets we put $B+B^{\prime}=\left\{\mathbf{k}+\mathbf{k}^{\prime}: \mathbf{k} \in B, \mathbf{k}^{\prime} \in B^{\prime}\right\}$. For every $\xi \in(0,1)$ and $\mathbf{0} \neq \mathbf{n} \in \mathbb{Z}^{d}$ we define cones

$$
\begin{aligned}
& C^{+}(\mathbf{n}, \xi)=\left\{\mathbf{m} \in \mathbb{Z}^{d}:\langle\mathbf{m}, \mathbf{n}\rangle \geq \xi\|\mathbf{m}\|\|\mathbf{n}\|\right\} \\
& C^{-}(\mathbf{n}, \xi)=\left\{\mathbf{m} \in \mathbb{Z}^{d}:\langle\mathbf{m}, \mathbf{n}\rangle \leq-\xi\|\mathbf{m}\|\|\mathbf{n}\|\right\}
\end{aligned}
$$

and set

$$
C(\mathbf{n}, \xi)=C^{+}(\mathbf{n}, \xi) \cup C^{-}(\mathbf{n}, \xi) .
$$

Definition 2.2. Let $\mathbf{0} \neq \mathbf{n} \in \mathbb{Z}^{d}$ and $\xi \in(0,1)$. The equivalence class $\Delta_{X}(\bar{x})$ of a point $\bar{x} \in X$ has $(\mathbf{n}, \xi)$-specification if $\Delta_{X}(\bar{x})$ is dense in $X$, and 
if there exist constants $s^{\prime} \geq 1, t^{\prime} \geq 0$ with the following property: if $r \geq 0$, and if $x, x^{\prime} \in \Delta_{X}(\bar{x})$ satisfy that $\pi_{\mathbf{B}\left(s^{\prime} r+t^{\prime}\right)}(x)=\pi_{\mathbf{B}\left(s^{\prime} r+t^{\prime}\right)}\left(x^{\prime}\right)$, then we can find a $y \in \Delta_{X}(\bar{x})$ with

$$
y_{\mathbf{m}}= \begin{cases}x_{\mathbf{m}} & \text { for every } \mathbf{m} \in C^{+}(\mathbf{n}, \xi)+\mathbf{B}(r), \\ x_{\mathbf{m}}^{\prime} & \text { for every } \mathbf{m} \in C^{-}(\mathbf{n}, \xi)+\mathbf{B}(r) .\end{cases}
$$

The equivalence class $\Delta_{X}(\bar{x})$ has $\mathbf{n}$-specification if it has $(\mathbf{n}, \xi)$-specification for some $\xi \in(0,1)$, and the Gibbs relation $\Delta_{X}$ has $\mathbf{n}$-specification if there exists a point $\bar{x} \in X$ whose equivalence class has $\mathbf{n}$-specification.

Remark 2.3. Let $A$ be a finite set, $d \geq 2, F \subset \mathbb{Z}^{d}$ a finite set, and $P \subset A^{F}$. Then we define a sft $X_{(F, P)} \subset A^{Z^{d}}$ by

$$
X_{(F, P)}=\left\{x \in A^{\mathbb{Z}^{d}}: \pi_{F} \cdot \sigma_{\mathbf{m}}(x) \in P \text { for every } \mathbf{m} \in \mathbb{Z}^{d}\right\} .
$$

If $E \subset \mathbb{Z}^{d}$ is a finite set we shall call an element $y \in A^{E}$ allowed if

$$
\pi_{F \cap(E+\mathbf{n})}(y) \in \pi_{F \cap(E+\mathbf{n})}(P)
$$

for every $\mathbf{n} \in \mathbb{Z}^{d}$. In general, if $y \in A^{E}$ is allowed, there need not exist an element $x \in X_{(F, P)}$ with $\pi_{E}(x)=y$.

We fix a sft $X=X_{(F, P)} \subset A^{\mathbb{Z}^{d}}$. If $X$ has trivial fundamental group in the sense of $[\mathbf{G e P r}]$, then $\Delta_{X}(x)$ has $(\mathbf{n}, \xi)$-specification for every $x \in X$, $\mathbf{0} \neq \mathbf{n} \in \mathbb{Z}^{d}$, and $\xi \in(0,1)$. Indeed, if $C^{\prime} \subset \mathbb{R}^{d}$ is a convex subset, $N \geq 1$, and if $\bar{C}^{\prime}(N)=\left\{\mathbf{t} \in \mathbb{R}^{d}: \min _{\mathbf{s} \in C}\|\mathbf{s}-\mathbf{t}\| \leq N\right\}, C=C^{\prime} \cap \mathbb{Z}^{d}, \bar{C}(N)=\bar{C}^{\prime}(N) \cap \mathbb{Z}^{d}$, and $\partial_{N}(C)=\bar{C}(N) \backslash C$, then the triviality of the fundamental group of $X$ essentially implies the following: if $N$ is large enough, and if $y \in A^{\partial_{N}(C)}$ is allowed, then there exists an allowed point $x \in A^{\tilde{C}(N)}$ such that $\pi_{\partial_{N}(C)}(x)=$ $y$. A brief glance at Definition 2.2 shows that this implies that $\Delta_{X}$ has the required specification property.

We end this section with a brief discussion of the connection between topological transitivity of $\Delta_{X}$ and the topological transitivity of $\sigma_{\mathbf{n}}$ for some given, nonzero $\mathbf{n} \in \mathbb{Z}^{d}$. Example 2.4 below shows that this connection is by no means automatic.

Example 2.4. Let $d=2, A=\{0,1\}, F=I^{2}=\{0,1\}^{2}$,

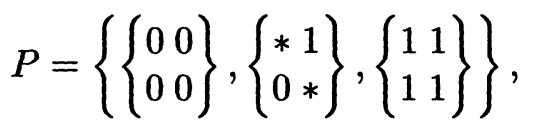

where $*$ indicates that either 0 or 1 can be put in that location, and let $X=X_{(F, P)}$. The equivalence relation $\Delta_{X}$ is topologically transitive, but not 
minimal, since $\Delta_{X}(\bar{y})=\{\bar{y}\}$ if $\bar{y}$ is one of the two fixed points of $\sigma=\sigma^{X}$, whereas $\Delta_{X}(\bar{x})$ has $(1,1)$-specification for $\bar{x}=\left(\bar{x}_{\mathbf{n}}\right)$ with

$$
\bar{x}_{\left(n_{1}, n_{2}\right)}= \begin{cases}0 & \text { if } n_{1}<-n_{2}, \\ 1 & \text { if } n_{1} \geq-n_{2} .\end{cases}
$$

The shift $\sigma_{(1,1)}$ is clearly not topologically transitive on $X$.

Let $A$ be a finite set, and let $X \subset A^{\mathbb{Z}^{d}}$ be a subshift. The $\mathbb{Z}^{d}$-action $\sigma^{X}$ is topologically mixing if there exists, for all nonempty open subsets $\mathcal{O}_{1}, \mathcal{O}_{2}$ of $X$, an integer $K \geq 1$ such that $\mathcal{O}_{1} \cap \sigma_{-\mathbf{n}}\left(\mathcal{O}_{2}\right) \neq \emptyset$ whenever $\|\mathbf{n}\| \geq K$. If we can find, for all nonempty, open subsets $\mathcal{O}_{1}, \ldots, \mathcal{O}_{r}$ of $X$, an integer $K \geq 1$ such that $\sigma_{-\mathbf{n}_{1}}\left(\mathcal{O}_{1}\right) \cap \cdots \cap \sigma_{-\mathbf{n}_{r}}\left(\mathcal{O}_{r}\right) \neq \emptyset$ whenever $\mathbf{n}_{1}, \ldots, \mathbf{n}_{r}$ are elements of $\mathbb{Z}^{d}$ with $\mathbf{n}_{1}=\mathbf{0}$ and $\left\|\mathbf{n}_{j}-\mathbf{n}_{i}\right\|>K$ for $1 \leq i<j \leq r$, then $\sigma^{X}$ is topologically mixing of order $r$. The action $\sigma^{X}$ is individually mixing if $\sigma_{\mathbf{n}}^{X}$ is topologically mixing for every nonzero $\mathbf{n} \in \mathbb{Z}^{d}$.

Lemma 2.5. Let $d \geq 2, A$ a finite set, and let $X \subset A^{\mathbb{Z}^{d}}$ be a sft. Suppose that there exists a periodic point $\bar{x} \in X$ for which $\Delta_{X}(\bar{x})$ is dense in $X$. Then $X$ has dpp, and $\sigma$ is topologically mixing of every order. In particular, $\sigma_{\mathbf{n}}$ is topologically transitive for every nonzero element $\mathbf{n} \in \mathbb{Z}^{d}$.

Proof. Fix $K \geq 1$ with $\sigma_{K \mathbf{m}}(\bar{x})=\bar{x}$ for every $\mathbf{m} \in \mathbb{Z}^{d}$. Since $\Delta_{X}(\bar{x})$ is dense in $X$ we can find, for every nonempty, closed and open subset $\mathcal{O} \subset X$, a point $x^{(\mathcal{O})} \in \Delta_{X}(\bar{x}) \cap \mathcal{O}$ and a positive integer $m^{(\mathcal{O})}$ with $\pi_{\mathbb{Z}^{d} \backslash \mathbf{B}\left(m^{(\mathcal{O})}\right)}\left(x^{(\mathcal{O})}\right)=$ $\pi_{\mathbb{Z}^{d} \backslash \mathbf{B}\left(m^{(\mathcal{O})}\right)}(\bar{x})$. The assertion about periodic points is obvious, since we can find, for each $x^{(\mathcal{O})}$, a periodic point $y^{(\mathcal{O})} \in X$ with $\pi_{\mathbf{B}(m(\mathcal{O})}\left(y^{(\mathcal{O})}\right)=$ $\pi_{\mathbf{B}\left(m^{(\mathcal{O})}\right)}\left(x^{(\mathcal{O})}\right)$.

In order to prove that $\sigma$ is topologically mixing of every order we fix $r \geq 2$ and nonempty, closed and open sets $\mathcal{O}_{1}, \ldots, \mathcal{O}_{r} \subset X$, and claim that $\sigma_{-\mathbf{n}_{1}}\left(\mathcal{O}_{1}\right) \cap \cdots \cap \sigma_{-\mathbf{n}_{r}}\left(\mathcal{O}_{r}\right) \neq \emptyset$ whenever $\mathbf{n}_{1}, \ldots, \mathbf{n}_{r}$ are elements of $\mathbb{Z}^{d}$ such that $\mathbf{n}_{1}=\mathbf{0}$ and $\left\|\mathbf{n}_{j}-\mathbf{n}_{i}\right\|$ is sufficiently large for all $i, j$ with $1 \leq i<j \leq r$. Let

$$
\begin{aligned}
W=\left\{\sigma_{-\mathbf{k}}\left(\mathcal{O}_{i}\right): 1 \leq i \leq r \text { and } \mathbf{k}=\left(k_{1}, \ldots, k_{d}\right) \in \mathbb{Z}^{d}\right. \text { with } \\
\left.0 \leq k_{j}<K \text { for } 1 \leq j \leq d\right\},
\end{aligned}
$$

and let $M=\max \left\{m^{(\mathcal{O})}: \mathcal{O} \in W\right\}$. If $\mathbf{n}_{1}=\mathbf{0}$ and $\left\|\mathbf{n}_{j}-\mathbf{n}_{i}\right\|>2 M$ for $1 \leq i<j \leq r$ there exists, for every choice of $\mathcal{O}_{i}^{\prime} \in W, i=1, \ldots, r$, a unique point $y \in \Delta_{X}(\bar{x})$ with

$$
\pi_{\mathbf{B}(M)}\left(\sigma_{-K \mathbf{n}_{j}}(y)\right)=\pi_{\mathbf{B}(M)}\left(\sigma_{-K \mathbf{n}_{j}}\left(x^{\left(\mathcal{O}_{j}^{\prime}\right)}\right)\right)
$$

for every $j=1, \ldots, r$, and with

$$
\pi_{\mathbb{Z}^{d} \backslash \bigcup \bigcup_{i=1}^{r} \mathbf{B}(M)+K \mathbf{n}_{i}}(y)=\pi_{\mathbb{Z}^{d} \backslash \bigcup_{i=1}^{r} \mathbf{B}(M)+K \mathbf{n}_{i}}(\bar{x}) .
$$


In particular, $\sigma_{-K \mathbf{n}_{1}}\left(\mathcal{O}_{1}^{\prime}\right) \cap \cdots \cap \sigma_{K \mathbf{n}_{r}\left(\mathcal{O}_{r}^{\prime}\right)} \neq \emptyset$ whenever $\mathbf{n}_{1}, \ldots, \mathbf{n}_{r} \in \mathbb{Z}^{d}$, $\mathbf{n}=\mathbf{0}$, and $\left\|\mathbf{n}_{j}-\mathbf{n}_{i}\right\|>2 K M$ for $1 \leq i<j \leq r$.

Since $\sigma$ is topologically mixing of every order, $\sigma_{\mathbf{n}}$ is topologically mixingand hence topologically transitive-for every nonzero element $\mathbf{n} \in \mathbb{Z}^{d}$.

\section{Cohomology.}

Let $A$ be a finite set, $d \geq 1, X \subset A^{\mathbb{Z}^{d}}$ a subshift, $G$ a locally compact, second countable group, and assume that $G$ has a metric $\gamma: G \times G \longmapsto \mathbb{R}^{+}$which is doubly invariant, i.e. which satisfies that $\gamma\left(g, g^{\prime}\right)=\gamma\left(h g, h g^{\prime}\right)=\gamma\left(g h, g^{\prime} h\right)$ for all $g, g^{\prime}, h \in G$ (such a metric exists, for example, if $G$ is compact, discrete, or abelian). The metric $\gamma$ will be fixed throughout the following discussion. As in [KaS] we set, for every continuous function $f: X \longmapsto G$ and every integer $r \geq 0$,

$$
\omega_{r}(f)=\sup _{\left\{\left(x, x^{\prime}\right) \in X \times X: \pi_{\mathbf{B}(r)}(x)=\pi_{\mathbf{B}(r)}\left(x^{\prime}\right)\right\}} \gamma\left(f(x), f\left(x^{\prime}\right)\right),
$$

and we say that $f$ has summable variation if

$$
\omega(f)=\sum_{r=1}^{\infty} \omega_{r}(f)<\infty
$$

The function $f$ if Hölder if there exist constants $\omega, \omega^{\prime}>0$ with $0<\omega<1$ such that

$$
\omega_{r}(f)<\omega^{\prime} \omega^{r}
$$

for every $r \geq 1$. If $G$ is discrete, for every continuous function $f: X \longmapsto G$ is Hölder.

A map $c: \mathbb{Z}^{d} \times X \longmapsto G$ is a continuous cocycle for the shift-action $\sigma$ of $\mathbb{Z}^{d}$ on $X$ defined in (2.1) if $c(\mathbf{n}, \cdot): X \longmapsto G$ is continuous for every $\mathbf{n} \in \mathbb{Z}^{d}$, and

$$
c(\mathbf{m}+\mathbf{n}, x)=c\left(\mathbf{m}, \sigma_{\mathbf{n}}(x)\right) \cdot c(\mathbf{n}, x)
$$

for all $x \in X$ and $\mathbf{m}, \mathbf{n} \in \mathbb{Z}^{d}$. The cocycle $c$ is a homomorphism if $c(\mathbf{n}, \cdot)$ is constant for every $\mathbf{n} \in \mathbb{Z}^{d}$, and $c$ is a coboundary if there exists a Borel map $b: X \longmapsto G$ such that

$$
c(\mathbf{n}, x)=b\left(\sigma_{\mathbf{n}}(x)\right)^{-1} \cdot b(x)
$$

for all $x \in X$ and $\mathbf{n} \in \mathbb{Z}^{d}$. The map $b$ in (3.5) is the cobounding function of $c$. Two cocycles $c, c^{\prime}: \mathbb{Z}^{d} \times X \longmapsto G$ are cohomologous, with transfer function 
$b$, if $c(\mathbf{n}, x)=b\left(\sigma_{\mathbf{n}}(x)\right)^{-1} \cdot c^{\prime}(\mathbf{n}, x) \cdot b(x)$ for all $\mathbf{n} \in \mathbb{Z}^{d}$ and $x \in X$. A cocycle $c: \mathbb{Z}^{d} \times X \longmapsto G$ has summable variation or is Hölder if $c(\mathbf{n}, \cdot)$ has summable variation or is Hölder for every $\mathbf{n} \in \mathbb{Z}^{d}$. Two cocycles $c, c^{\prime}: \mathbb{Z}^{d} \times X \longmapsto G$ are continuously (or Hölder) cohomologous if they are cohomologous with continuous (or Hölder) transfer function.

If $\mathbf{R} \subset X \times X$ is an equivalence relation we call a Borel map $c: \mathbf{R} \longmapsto G$ a cocycle if

$$
c\left(x, x^{\prime \prime}\right)=c\left(x, x^{\prime}\right) \cdot c\left(x^{\prime}, x^{\prime \prime}\right)
$$

for all $x, x^{\prime}, x^{\prime \prime} \in X$ with $\left(x, x^{\prime}\right),\left(x, x^{\prime \prime}\right) \in \mathbf{R}$. Two cocycles $c_{1}, c_{2}: \mathbf{R} \longmapsto G$ are cohomologous if there exists a Borel map $b: X \longmapsto G$ with

$$
c_{1}\left(x, x^{\prime}\right)=b(x)^{-1} \cdot c_{2}\left(x, x^{\prime}\right) \cdot b\left(x^{\prime}\right)
$$

for all $\left(x, x^{\prime}\right) \in \mathbf{R}$, and a cocycle $c$ is a coboundary if it is cohomologous to the constant cocycle $c_{0}$ defined by $c_{0}\left(x, x^{\prime}\right)=1$ for all $\left(x, x^{\prime}\right) \in \mathbf{R}$, where $1=1_{G}$ is the identity element of $G$.

Suppose that $d \geq 1, A$ a finite set, $X \subset A^{\mathbb{Z}^{d}}$ a subshift, and $f: X \longmapsto G$ a function with summable variation. We define cocycles $c_{f}^{(\mathbf{n}, \pm)}: \Delta_{X} \longmapsto G$ by setting

$$
\begin{aligned}
& c_{f}^{(\mathbf{n},+)}\left(x, x^{\prime}\right)=\left(\prod_{k=0}^{\infty} f\left(\sigma_{k \mathbf{n}}(x)\right)^{-1}\right) \cdot\left(\prod_{k=0}^{\infty} f\left(\sigma_{k \mathbf{n}}\left(x^{\prime}\right)\right)^{-1}\right)^{-1}, \\
& c_{f}^{(\mathbf{n},-)}\left(x, x^{\prime}\right)=\left(\prod_{k=1}^{\infty} f\left(\sigma_{-k \mathbf{n}}(x)\right)\right) \cdot\left(\prod_{k=1}^{\infty} f\left(\sigma_{-k \mathbf{n}}\left(x^{\prime}\right)\right)\right)^{-1},
\end{aligned}
$$

for every $\left(x, x^{\prime}\right) \in \Delta_{X}$. In order to explain the notation in (3.8) and check that these cocycles are well defined we assume for the moment that the function $f$ only depends on finitely many coordinates and choose an integer $N \geq 0$ such that $f(x)=f\left(x^{\prime}\right)$ whenever $x, x^{\prime} \in X$ and $\pi_{\mathbf{B}(N)}(x)=\pi_{\mathbf{B}(N)}\left(x^{\prime}\right)$. For every $\left(x, x^{\prime}\right) \in \Delta_{X}$ there exists an integer $M \geq 0$ such that $x_{\mathbf{m}}=x_{\mathbf{m}}^{\prime}$ for all $\mathbf{m} \in \mathbb{Z}^{d}$ with $\|\mathbf{m}\| \geq M$, and our choice of $N$ implies that $f \cdot \sigma_{k \mathbf{n}}(x)=$ $f \cdot \sigma_{k \mathbf{n}}\left(x^{\prime}\right)$ for all $k \in \mathbb{Z}$ with $|k|>M+N$. Then

$$
\begin{aligned}
c_{f}^{(\mathbf{n},+)}\left(x, x^{\prime}\right)= & \left(\prod_{k=0}^{\infty} f\left(\sigma_{k \mathbf{n}}(x)\right)^{-1}\right) \cdot\left(\prod_{k=0}^{\infty} f\left(\sigma_{k \mathbf{n}}\left(x^{\prime}\right)\right)^{-1}\right)^{-1} \\
= & f(x)^{-1} \cdots f\left(\sigma_{(M+N) \mathbf{n}}(x)\right)^{-1} \\
& \cdot f\left(\sigma_{(M+N) \mathbf{n}}\left(x^{\prime}\right)\right) \cdots f\left(x^{\prime}\right),
\end{aligned}
$$

since all other terms in the infinite product are deemed to cancel. If $f$ has summable variation, our assumption that $G$ possesses a doubly invariant 
metric implies that the finite products

$$
f(x)^{-1} \cdots f\left(\sigma_{m \mathbf{n}}(x)\right)^{-1} \cdot f\left(\sigma_{m \mathbf{n}}\left(x^{\prime}\right)\right) \cdots f\left(x^{\prime}\right)
$$

converge to $c_{f}^{(\mathbf{n},+)}\left(x, x^{\prime}\right)$ for all $\left(x, x^{\prime}\right) \in \Delta_{X}$ as $m \rightarrow \infty$, so that $c_{f}^{(\mathbf{n},+)}$ is again well defined. The existence of $c_{f}^{(\mathbf{n},-)}$ is verified in exactly the same manner.

Proposition 3.1. Let $d \geq 1, A$ a finite set, $X \subset A^{\mathbb{Z}^{d}}$ a subshift, $G$ a locally compact, second countable group with a fixed doubly invariant metric $\gamma$, and $f: X \longmapsto G$ a function with summable variation. Suppose that the Gibbs equivalence class $\Delta_{X}(\bar{x})$ has $(\mathbf{n}, \xi)$-specification for some $\bar{x} \in X$, some nonzero $\mathbf{n} \in \mathbb{Z}^{d}$, and some $\xi \in(0,1)$, and that the cocycles $c_{f}^{(\mathbf{n}, \pm)}: \Delta_{X} \longmapsto G$ in (3.8) are equal. Then there exists a continuous function $b: X \longmapsto G$ such that the map $x \longmapsto b\left(\sigma_{\mathbf{n}}(x)\right)^{-1} \cdot f(x) \cdot b(x)$ is constant on $X$. If $f$ is Hölder, the function $b: X \longmapsto G$ is again Hölder.

Proof. In order to simplify notation we set $c^{ \pm}=c_{f}^{(\mathbf{n}, \pm)}$. For every $r>0$ we put

$$
\begin{aligned}
& \Delta^{+}(r)=\left\{\left(x, x^{\prime}\right) \in \Delta_{X}: \pi_{C^{-}(\mathbf{n}, \xi)+\mathbf{B}(r)}(x)=\pi_{C^{-}(\mathbf{n}, \xi)+\mathbf{B}(r)}\left(x^{\prime}\right)\right\} \\
& \Delta^{-}(r)=\left\{\left(x, x^{\prime}\right) \in \Delta_{X}: \pi_{C^{+}(\mathbf{n}, \xi)+\mathbf{B}(r)}(x)=\pi_{C^{+}(\mathbf{n}, \xi)+\mathbf{B}(r)}\left(x^{\prime}\right)\right\}
\end{aligned}
$$

and note that $\gamma\left(c^{ \pm}\left(x, x^{\prime}\right), 1_{G}\right) \leq\left(1-\xi^{2}\right)^{-\frac{1}{2}} \cdot \sum_{k \geq r} \omega_{k}(f)<\infty$ for all $\left(x, x^{\prime}\right) \in$ $\Delta^{\mp}(r)$.

The $(\mathbf{n}, \xi)$-specification of $\Delta_{X}(\bar{x})$ allows us to choose constants $s^{\prime} \geq 1$, $k^{\prime} \geq 0$ such that we can find, for every $r \geq 0$, and for all $x, x^{\prime} \in \Delta_{X}(\bar{x})$ with $\pi_{\mathbf{B}\left(s^{\prime} r+t^{\prime}\right)}(x)=\pi_{\mathbf{B}\left(s^{\prime} r+t^{\prime}\right)}\left(x^{\prime}\right)$, an element $y \in \Delta_{X}(\bar{x})$ with $(x, y) \in \Delta^{+}(r)$ and $\left(x^{\prime}, y\right) \in \Delta^{-}(r)$. Since the metric $\gamma$ is doubly invariant and $c^{+}=c^{-}$we obtain that

$$
\begin{gathered}
\gamma\left(c^{+}\left(x, x^{\prime}\right), c^{+}(x, y)\right) \leq\left(1-\xi^{2}\right)^{-\frac{1}{2}} \sum_{k \geq r} \omega_{k}(f)=C^{\prime}(r) \\
\gamma\left(c^{+}(x, y), 1_{G}\right)=\gamma\left(c^{-}(x, y), 1_{G}\right) \leq C^{\prime}(r) \text { and hence } \\
\gamma\left(c^{+}\left(x, x^{\prime}\right), 1_{G}\right) \leq 2 C^{\prime}(r)
\end{gathered}
$$

for all $x, x^{\prime} \in \Delta_{X}(\bar{x})$ with $\pi_{\mathbf{B}\left(s^{\prime} r+t^{\prime}\right)}(x)=\pi_{\mathbf{B}\left(s^{\prime} r+t^{\prime}\right)}\left(x^{\prime}\right)$. By varying $r$ we see that

$$
\lim _{\substack{\left.x, x^{\prime}\right) \rightarrow \Delta \\ x^{\prime} \in \Delta_{X}(\bar{x})}} \gamma\left(c^{+}\left(x, x^{\prime}\right), 1_{G}\right)=0
$$

where $\Delta=\{(x, x): x \in X\} \subset X \times X$. The cocycle equation (3.6) shows that the function $c^{+}(\cdot, \cdot): \Delta_{X}(\bar{x}) \times \Delta_{X}(\bar{x}) \longmapsto G$ has a unique extension to 
a continuous function $\bar{c}^{+}: X \times X \longmapsto G$ with $\bar{c}^{+}\left(x, x^{\prime}\right)=c^{+}\left(x, x^{\prime}\right)$ for all $x, x^{\prime} \in \Delta_{X}(\bar{x})$, and $\bar{c}^{+}$is a cocycle on the equivalence relation $\mathbf{R}=X \times X$. We define $b: X \longmapsto G$ by $b(x)=\bar{c}^{+}(x, \bar{x})$ for every $x \in X$, and obtain that $\bar{c}^{+}\left(x, x^{\prime}\right)=b(x) \cdot b\left(x^{\prime}\right)^{-1}$ for all $x, x^{\prime} \in X$.

If $f$ is Hölder we choose $0<\omega<1, \omega^{\prime}>0$, such that (3.3) is satisfied. From (3.9) we see that there exists a positive constant $\bar{\omega}^{\prime}$ such that $\gamma\left(c^{+}\left(x, x^{\prime}\right), 1_{G}\right) \leq \bar{\omega}^{\prime} \omega^{r}$ for all $\left(x, x^{\prime}\right) \in \Delta_{X}\left(x_{0}\right)$ with $\pi_{\mathbf{B}\left(s^{\prime} r+t^{\prime}\right)}(x)=$ $\pi_{\mathbf{B}\left(s^{\prime} r+t^{\prime}\right)}\left(x^{\prime}\right)$, and we conclude that $\gamma\left(\bar{c}^{+}\left(x, x^{\prime}\right), 1_{G}\right) \leq \bar{\omega}^{\prime} \omega^{r}$ for all $\left(x, x^{\prime}\right)$ $\in X \times X$ with $\pi_{\mathbf{B}\left(s^{\prime} r+t^{\prime}\right)}(x)=\pi_{\mathbf{B}\left(s^{\prime} r+t^{\prime}\right)}\left(x^{\prime}\right)$. Hence $\gamma\left(b(x), b\left(x^{\prime}\right)\right) \leq 2 \bar{\omega}^{\prime} \omega^{r}$ whenever $\pi_{\mathbf{B}\left(s^{\prime} r+t^{\prime}\right)}(x)=\pi_{\mathbf{B}\left(s^{\prime} r+t^{\prime}\right)}\left(x^{\prime}\right)$, which implies that $b$ is Hölder.

Define a second equivalence relation $\bar{\Delta}_{X}(\mathbf{n})$ on $X$ by setting

$$
\bar{\Delta}_{X}(\mathbf{n})=\left\{\left(x, \sigma_{k \mathbf{n}}\left(x^{\prime}\right)\right): k \in \mathbb{Z},\left(x, x^{\prime}\right) \in \Delta_{X}\right\},
$$

and consider the Borel map $\psi: \bar{\Delta}_{X}(\mathbf{n}) \longmapsto G$ given by

$$
\psi\left(x, \sigma_{k \mathbf{n}}\left(x^{\prime}\right)\right)=\left(\prod_{j=0}^{\infty} f\left(\sigma_{j \mathbf{n}}(x)\right)^{-1}\right) \cdot\left(\prod_{j=0}^{\infty} f\left(\sigma_{j \mathbf{n}}\left(x^{\prime}\right)\right)^{-1}\right)
$$

for every $\left(x, x^{\prime}\right) \in \Delta_{X}$ and $k \in \mathbb{Z}$, where we are using the same interpretation of the infinite products as in (3.8). Then $\psi$ is a cocycle on $\bar{\Delta}_{X}(\mathbf{n})$. In particular,

$$
\begin{aligned}
b(x) \cdot b\left(x^{\prime}\right)^{-1} & =\psi\left(x, x^{\prime}\right) \\
& =\psi\left(x, \sigma_{\mathbf{n}}(x)\right) \cdot \psi\left(\sigma_{\mathbf{n}}(x), \sigma_{\mathbf{n}}\left(x^{\prime}\right)\right) \cdot \psi\left(\sigma_{\mathbf{n}}\left(x^{\prime}\right), x^{\prime}\right) \\
& =f(x)^{-1} \cdot b\left(\sigma_{\mathbf{n}}(x)\right) \cdot b\left(\sigma_{\mathbf{n}}\left(x^{\prime}\right)\right)^{-1} \cdot f\left(x^{\prime}\right)
\end{aligned}
$$

for all $\left(x, x^{\prime}\right) \in \Delta_{X}$, so that $b\left(\sigma_{\mathbf{n}}(\cdot)\right)^{-1} \cdot f \cdot b$ is invariant under $\Delta_{X}$ (a map $\phi: X \longmapsto G$ is invariant under $\Delta_{X}$ if $\phi(x)=\phi\left(x^{\prime}\right)$ for every $\left.\left(x, x^{\prime}\right) \in \Delta_{X}\right)$. Since $\Delta_{X}$ is topologically transitive, the continuity of $f$ and $b$ yields that $b\left(\sigma_{\mathbf{n}}(\cdot)\right)^{-1} \cdot f \cdot b$ is constant on $X$.

Theorem 3.2. Let $d \geq 2, A$ a finite set, $X \subset A^{\mathbb{Z}^{d}}$ a subshift, and $G$ a locally compact, second countable group with a fixed doubly invariant metric $\gamma$. We write $\sigma$ for the shift-action (2.1) on $X$ and assume that there exists a nonzero element $\mathbf{n} \in \mathbb{Z}^{d}$ such that the Gibbs equivalence relation $\Delta_{X}$ has $\mathbf{n}$-specification and $\sigma_{\mathbf{n}}$ is topologically mixing. Then every cocycle $c: \mathbb{Z}^{d} \times$ $X \longmapsto G$ with summable variation is cohomologous to a homomorphism, with continuous transfer function. If $c$ is Hölder, the transfer function is again Hölder.

Proof. Suppose that $c: \mathbb{Z}^{d} \times X \longmapsto G$ is a cocycle with summable variation. We set $f=c(\mathbf{n}, \cdot)$ and define the $\operatorname{cocycles} c_{f}^{(\mathbf{n}, \pm)}: \Delta_{X} \longmapsto G$ by (3.8). From 
the cocycle equation (3.4) we know that $c\left(\mathbf{m}, \sigma_{\mathbf{n}}(x)\right) \cdot f(x) \cdot c(\mathbf{m}, x)^{-1}=$ $f\left(\sigma_{\mathbf{m}}(x)\right)$ for all $\mathbf{m} \in \mathbb{Z}^{d}$ and $x \in X$, and we conclude that $c\left(\mathbf{m}, \sigma_{(k+1) \mathbf{n}}(x)\right)$. $f\left(\sigma_{k \mathbf{n}}(x)\right) \cdot c\left(\mathbf{m}, \sigma_{k \mathbf{n}}(x)\right)^{-1}=f\left(\sigma_{\mathbf{m}+k \mathbf{n}}(x)\right)$ for all $k \in \mathbb{Z}, \mathbf{m} \in \mathbb{Z}^{d}$, and $x \in$ $X$, so that $c_{f}^{(\mathbf{n}, \pm)}\left(x, x^{\prime}\right)=c(\mathbf{m}, x) \cdot c_{f}^{(\mathbf{n}, \pm)}\left(\sigma_{\mathbf{m}}(x), \sigma_{\mathbf{m}}\left(x^{\prime}\right)\right) \cdot c\left(\mathbf{m}, x^{\prime}\right)^{-1}$ for all $\left(x, x^{\prime}\right) \in \Delta_{X}$ and $\mathbf{m} \in \mathbb{Z}^{d}$. Since $f$ has summable variation we obtain that

$$
\begin{aligned}
\gamma\left(c_{f}^{(\mathbf{n},+)}\left(x, x^{\prime}\right), c_{f}^{(\mathbf{n},-)}\left(x, x^{\prime}\right)\right) \\
\quad=\lim _{l \longmapsto \infty} \gamma\left(c_{f}^{(\mathbf{n},+)}\left(\sigma_{l \mathbf{m}}(x), \sigma_{l \mathbf{m}}\left(x^{\prime}\right)\right), c_{f}^{(\mathbf{n},-)}\left(\sigma_{l \mathbf{m}}(x), \sigma_{l \mathbf{m}}\left(x^{\prime}\right)\right)\right)=0
\end{aligned}
$$

for all $\left(x, x^{\prime}\right) \in \Delta_{X}$ and $\mathbf{0} \neq \mathbf{m} \in \mathbb{Z}^{d}$ with $\langle\mathbf{m}, \mathbf{n}\rangle=0$. According to Proposition 3.1 this implies that there exists a continuous (or Hölder) function $b: X \longmapsto G$ such that the map $x \longmapsto b\left(\sigma_{\mathbf{n}}(x)\right)^{-1} \cdot c(\mathbf{n}, x) \cdot b(x)$ is everywhere equal to some element $a \in G$, and we define a cocycle $c^{\prime}: \mathbb{Z}^{d} \times X \longmapsto G$ by setting $c^{\prime}(\mathbf{m}, x)=b\left(\sigma_{\mathbf{m}}(x)\right)^{-1} \cdot c(\mathbf{m}, x) \cdot b(x)$ for every $\mathbf{m} \in \mathbb{Z}^{d}$ and $x \in X$. Then $c^{\prime}$ is cohomologous to $c$, and the cocycle equation (3.4) shows that

$$
c^{\prime}(\mathbf{m}, x)=a^{-k} \cdot c^{\prime}\left(\mathbf{m}, \sigma_{k \mathbf{n}}(x)\right) \cdot a^{k}
$$

for every $\mathbf{m} \in \mathbb{Z}^{d}, k \in \mathbb{Z}$, and $x \in X$. We fix $\mathbf{m} \in \mathbb{Z}^{d}$ and set $g=c^{\prime}(\mathbf{m}, \cdot)$. If $g$ is not constant, then we can find $\varepsilon>0, g_{1}, g_{2} \in G$, and nonempty, open sets $\mathcal{O}_{1}$ and $\mathcal{O}_{2}$, such that $\sup _{x \in \mathcal{O}_{i}} \gamma\left(g(x), g_{i}\right)<\varepsilon$ and $\gamma\left(g_{1}, g_{2}\right)>3 \varepsilon$. From (3.10) we see that $\gamma\left(a^{-k} \cdot g(y) \cdot a^{k}, g_{1}\right)<\varepsilon$ for every $k \in \mathbb{Z}$ and $y \in \sigma_{-k \mathbf{n}}\left(\mathcal{O}_{1}\right)$. However, since $\sigma_{\mathbf{n}}$ is topologically mixing, we know that $\sigma_{k \mathbf{n}}\left(\mathcal{O}_{1}\right) \cap \mathcal{O}_{i} \neq \emptyset$ for $i=1,2$, and for all sufficiently large $k$. If $k>0$ is large enough we can thus find elements $y_{1}, y_{2} \in \sigma_{-k \mathbf{n}}\left(\mathcal{O}_{1}\right)$ such that $\gamma\left(g\left(y_{i}\right), g_{i}\right)<\varepsilon$ for $i=1,2$. This violates the invariance of the metric $\gamma$ and implies that $c^{\prime}(\mathbf{m}, \cdot)$ must be constant. As $\mathbf{m} \in \mathbb{Z}^{d}$ was arbitrary, $c^{\prime}$ is a homomorphism.

Corollary 3.3. Assume that $X \subset A^{\mathbb{Z}^{d}}$ is $\mathrm{sft}$, and that there exists a periodic point $\bar{x} \in X$ such that $\Delta_{X}(\bar{x})$ has $\mathbf{n}$-specification for some nonzero $\mathbf{n} \in \mathbb{Z}^{d}$. If $G$ is a locally compact, second countable group with a fixed doubly invariant metric $\gamma$ then every cocycle $c: \mathbb{Z}^{d} \times X \longmapsto G$ with summable variation is cohomologous to a homomorphism, with continuous transfer function. If $c$ is Hölder, the transfer function is again Hölder.

Proof. Theorem 3.2 and Lemma 2.5 .

Corollary 3.4. Let $d \geq 2, A$ a finite set, $X \subset A^{\mathbb{Z}^{d}} a$ sft which satisfies the conditions of Corollary 3.3, and let $\sigma$ be the shift-action of $\mathbb{Z}^{d}$ on $X$. Assume that $G \neq\{0\}$ is a countable discrete group, $H \subsetneq G$ a subgroup, and $c: \mathbb{Z}^{d} \times X \longmapsto G$ a continuous cocycle, and denote by $\sigma^{(c, H)}$ the skew-product action of $\mathbb{Z}^{d}$ on $X \times G / H$ defined by

$$
\sigma_{\mathbf{n}}^{(c, H)}(x, g H)=\left(\sigma_{\mathbf{n}}(x), c(\mathbf{n}, x) \cdot g H\right)
$$


for every $\mathbf{n} \in \mathbb{Z}^{d}, x \in X$, and $g \in G$. Then $\sigma_{\mathbf{n}}^{(c, H)}$ is topologically nonmixing for every $\mathbf{n} \in \mathbb{Z}^{d}$.

Proof. Corollary 3.3 implies that $c$ is continuously cohomologous to a homomorphism, and we assume without loss in generality that $c$ is a homomorphism. Then $\sigma_{\mathbf{n}}^{(c, H)}$ is obviously nonmixing for every $\mathbf{n} \in \mathbb{Z}^{d}$.

Corollary 3.5. Let $d \geq 2, A$ a finite set, $X \subset A^{\mathbf{Z}^{d}} a$ sft which satisfies the conditions of Corollary 3.3, and let $\sigma$ be the shift-action of $\mathbb{Z}^{d}$ on $X$. If $\sigma$ is individually mixing then there does not exist a finite set $B, a \mathrm{sft}$ $Y \subset B^{Z^{d}}$ such that the shift-action $\sigma^{Y}$ of $\mathbb{Z}^{d}$ on $Y$ is individually mixing, and a continuous, shift-commuting, open map $\phi: Y \longmapsto X$ which is everywhere $n$-to-1 for some $n \geq 2$.

Proof. If there exists a shift-commuting, open map $\phi: Y \longmapsto X$ which is everywhere $n$-to- 1 for some $n \geq 2$, then [Mic] allows us to find a homeomorphism $\psi: Y^{\prime}=X \times\{1, \ldots, n\} \longmapsto Y$ with $\phi \cdot \psi(x, k)=x$ for every $(x, k) \in Y^{\prime}$. Since $\phi \cdot \sigma_{\mathbf{n}}^{Y}=\sigma_{\mathbf{n}} \cdot \phi$ for every $\mathbf{n} \in \mathbb{Z}^{d}$, there exists a continuous cocycle $c: \mathbb{Z}^{d} \times X \longmapsto \mathcal{S}_{n}$ with values in the symmetric group $\mathcal{S}_{n}$ on $\{1, \ldots, n\}$ such that $\psi\left(\sigma_{\mathbf{n}}(x), c(\mathbf{n}, x)(k)\right)=\sigma_{\mathbf{n}}^{Y} \cdot \psi(x, k)$ for every $\mathbf{n} \in \mathbb{Z}^{d}$ and $(x, k) \in Y^{\prime}$. The proof is completed by setting $H$ equal to the stability subgroup of the symbol 1 in $G=\mathcal{S}_{n}$, and by applying Corollary 3.4.

Corollary 3.6. Let $d \geq 2, A$ a finite set, $X \subset A^{\mathbf{Z}^{d}} a$ sft, and assume that there exists a periodic point $\bar{x} \in X$ such that $\Delta_{X}(\bar{x})$ is dense in $X$, and that $X$ has trivial fundamental group in the sense of $[\mathbf{G e P r}]$. If $\sigma$ is the shiftaction of $\mathbb{Z}^{d}$ on $X$ and $G$ a locally compact, second countable group with a fixed doubly invariant metric $\gamma$, then every cocycle $c: \mathbb{Z}^{d} \times X \longmapsto G$ with summable variation is cohomologous to a homomorphism, with continuous transfer function. If $c$ is Hölder, then the transfer function is again Hölder.

Proof. According to Remark 2.3, the triviality of the fundamental group of $X$ implies that $X$ satisfies the hypotheses of Corollary 3.3.

\section{Examples with positive entropy.}

In this section we consider some of higher-dimensional sft's with positive entropy and discuss their connections with Corollary 3.3. If $A$ is a finite set, $d \geq 2, F \subset \mathbb{Z}^{d}$ a finite set, and $P \subset A^{F}$, then we define the sft $X_{(F, P)} \subset A^{\mathbb{Z}^{d}}$ by $(2.10)$.

4.1. The Full Shift. Let $A$ be a finite set, and let $X=A^{\mathbb{Z}^{d}}$ be the full $d$ dimensional $|A|$-shift. Then $X$ is a sft, $\Delta_{X}(\bar{x})$ has $(\mathbf{n}, \xi)$-specification whenever $\bar{x} \in X$ is a fixed point (or, indeed, any other point), $\mathbf{0} \neq \mathbf{n} \in \mathbb{Z}^{d}$, and 
$\xi \in(0,1)$. Hence Corollary 3.3 applies. A special case of this result is due to J. Kammeyer ([Kam1], [Kam2]).

4.2. Dimers on the Square Lattice. Let $d=2, A=\{0,1,2,3\}$,

$$
F=\left\{\begin{array}{l}
(0,1) \\
(0,0) \\
(1,0)
\end{array}\right\}
$$

and

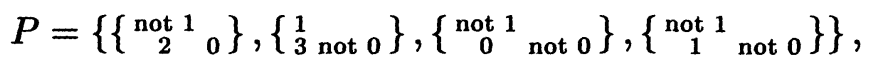

where 'not $j$ ' means that we can put any symbol other than $j$ in this location. The sft $X=X_{(F, P)}$ is isomorphic to the set of all tilings of $\mathbb{Z}^{2}$ by dominoes or dimers, where each domino covers exactly two horizontally or vertically adjacent lattice points (cf. [Kas], [TeL], [Sch2]): the isomorphism is obtained by interpreting 0 and 2 as the right and left endpoints of a horizontal domino, and 1 and 3 as the top and bottom endpoints of a vertical domino. It is easy to see that $\Delta_{X}$ is topologically transitive, but not minimal: for example, $\Delta_{X}(\bar{y})=\{\bar{y}\}$ for the periodic point

$$
\begin{aligned}
& .202020202 \text {. } \\
& .020202020 \text {. }
\end{aligned}
$$

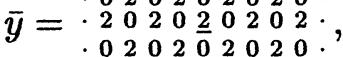

$$
\begin{aligned}
& .202020202 \text {. }
\end{aligned}
$$

whereas $\Delta_{X}(\bar{x})$ is dense in $X$ for the periodic point

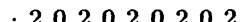

$$
\begin{aligned}
& \begin{array}{lllllllll}
2 & 0 & 2 & 0 & 2 & 0 & 2 & 0 & 2
\end{array} \text {. }
\end{aligned}
$$

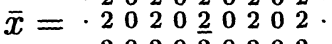

$$
\begin{aligned}
& \text { - } 20200200202 \text {. } \\
& 202020202 \text {. }
\end{aligned}
$$

where the 0-coordinates of $\bar{x}$ and $\bar{y}$ are underlined. In order to see that $\Delta_{X}(\bar{x})$ is indeed dense in $X$ we fix an even integer $N \geq 2$ and an element $y \in X$, put $Q(N)=\{-N, \ldots, N\}^{d} \subset \mathbb{Z}^{d}$ and $z=\pi_{Q(N)}(y) \in \pi_{Q(N)}(X) \subset A^{Q(N)}$, and set, for every $M \geq N, S^{+}(N, M)=\{-N, \ldots, N\} \times\{-N, \ldots, M\}$. If $z$ has been extended to an element of $\pi_{S^{+}(N, M)}(X)$ for some $M \geq N$ we set

$$
z_{(-N, M+1)}= \begin{cases}2 \quad \text { if } z_{(k, M)} \neq 3 \text { for all } k \in\{-N, \ldots, N\} \\ \quad \text { or if } \min \left\{k \in\{-N, \ldots, N\}: z_{(k, M)}=3\right\} \text { is even } \\ 1 \quad \text { if } z_{(-N, M)}=3 \\ 0 \quad \text { otherwise }\end{cases}
$$

and define $z_{(k, M+1)}$ successively for $k=-N+1, \ldots, N$ by setting $z_{(k, M+1)}=$ 2 whenever possible. This definition of $z$ has the property that the number of 
coordinates $\left\{k \in\{-N, \ldots, N\}: z_{(k, M)}=2\right\}$ is a strictly decreasing function of $M$, and we conclude the existence of an integer $M^{+} \geq N$ such that

$$
z_{\left(k, M^{+}\right)}= \begin{cases}2 & \text { if } k \in\{-N, \ldots, N\} \text { is even } \\ 0 & \text { if } k \in\{-N, \ldots, N\} \text { is odd }\end{cases}
$$

Put $Q=\{-N, \ldots, N\} \times\left\{-N, \ldots, M^{+}\right\}$, and apply an analogous argument to the left vertical edge of the rectangle $Q$. In doing this we may occasionally have to add a horizontal row at the top of $Q$ whose coordinates are all in $\{0,1,2\}$; if we have to add such a row we can add a second row with all coordinates in $\{0,2\}$, starting with ' 2 ' as we move from left to right. By repeating this process we eventually obtain a rectangle $Q^{\prime}=\left\{-N^{\prime}, \ldots, N\right\} \times$ $\left\{-N, \ldots, M^{\prime}\right\}$ and a point $z^{\prime} \in \pi_{Q^{\prime}}(X)$ such that $\pi_{Q}\left(z^{\prime}\right)=z$, all coordinates of $z^{\prime}$ along the top row of $Q^{\prime}$ lie in $\{0,2\}$, starting with ' 2 ' as we move from left to right, and $z_{\left(m,-N^{\prime}\right)}^{\prime} \in\{1,3\}$ for all $m \in\left\{-N, \ldots, M^{\prime}-1\right\}$. By adding yet another column and row on the left and at the top of $Q^{\prime}$ and thereby increasing $M^{\prime}$ and $N^{\prime}$ by 1 , if necessary, we may assume in addition that $N^{\prime}$ is even,

$$
z_{\left(k, M^{\prime}\right)}= \begin{cases}2 & \text { if } k \in\left\{-N^{\prime}, \ldots, N\right\} \text { is even, } \\ 0 & \text { if } k \in\left\{-N^{\prime}, \ldots, N\right\} \text { is odd, }\end{cases}
$$

and $z_{\left(-N^{\prime}, k\right)} \in\{1,3\}$ for all $k \in\left\{-N, \ldots, M^{\prime}-1\right\}$. Next we set $Q^{\prime \prime}=$ $\left\{-N^{\prime}, \ldots, 2 N+N^{\prime}+1\right\} \times\left\{-N, \ldots, M^{\prime}\right\}$ and use 'reflection' to extend $z^{\prime}$ to point $z^{\prime \prime} \in \pi_{Q^{\prime \prime}}(X)$ as follows: for every $(k, l) \in Q^{\prime}$ we put $z_{(k, l)}^{\prime \prime}=z_{(k, l)}^{\prime}$ and

$$
z_{(2 N-k+1, l)}^{\prime \prime}= \begin{cases}z_{(k, l)}^{\prime} & \text { if } z_{(k, l)} \in\{1,3\} \\ 2 & \text { if } z_{(k, l)}^{\prime}=0, \\ 0 & \text { if } z_{(k, l)}^{\prime}=2 .\end{cases}
$$

Then $z^{\prime \prime}$ has ' 0 '-s nd ' 2 '-s along the top row, and ' 1 '-s and ' 3 '-s along the left and right vertical sides below the top corners of $Q^{\prime \prime}$. Finally we reflect $Q^{\prime \prime}$ and $z^{\prime \prime}$ vertically to obtain the rectangle $Q^{*}=\left\{-N^{\prime}, \ldots, 2 N+N^{\prime}+1\right\} \times$ $\left\{-2 N-M^{\prime}-1, \ldots, M^{\prime}\right\}$ and the point $z^{*} \in \pi_{Q^{*}}(X)$ given by $z_{(k, l)}^{*}=z_{(k, l)}^{\prime \prime}$ and

$$
z_{(k,-2 N-l-1)}^{*}= \begin{cases}z_{(k, l)}^{\prime \prime} & \text { if } z_{(k, l)} \in\{0,2\} \\ 1 & \text { if } z_{(k, l)}^{\prime \prime}=3, \\ 3 & \text { if } z_{(k, l)}^{\prime \prime}=1,\end{cases}
$$

for every $(k, l) \in Q^{\prime}$. The point $x^{*}$ has ' 0 '-s and ' 2 '-s along the top and bottom rows, and ' 1 '-s and ' 3 '-s along the left and right vertical sides (with the exception of the corners) of $Q^{*}$. Our construction guarantees the existence of a point $v \in \Delta_{X}(\bar{x})$ with $\pi_{Q^{*}}(v)=z^{*}$ and $\pi_{Q(N)}(v)=\pi_{Q(N)}(y)$. 
This shows that $\Delta_{X}$ is topologically transitive. However, Theorem 3.2 cannot be applied to $X$; indeed, if $G$ is the discrete matrix group $G=$ $\left\{\left(\begin{array}{cc} \pm 1 & k \\ 0 & 1\end{array}\right): k \in \mathbb{Z}\right\}$, then the shift-action $\sigma$ of $\mathbb{Z}^{d}$ on $X$ admits a Hölder cocycle $c: \mathbb{Z}^{d} \times X \longmapsto G$ which is not cohomologous to a homomorphism. This cocycle is related to the 'height-function' of domino-tilings defined in [Thu], and is given as follows:

$$
\begin{aligned}
& c((1,0), x)= \begin{cases}\left(\begin{array}{rr}
-1 & 1 \\
0 & 1
\end{array}\right) & \text { if } x_{(0,0)} \in\{0,2,3\}, \\
\left(\begin{array}{rl}
-1 & 3 \\
0 & 1
\end{array}\right) & \text { if } x_{(0,0)}=1,\end{cases} \\
& c((0,1), x)=\left\{\begin{aligned}
\left(\begin{array}{rl}
-1 & 2 \\
0 & 1
\end{array}\right) & \text { if } x_{(0,0)} \in\{1,2,3\}, \\
\left.\begin{array}{rr}
-1 & 0 \\
0 & 1
\end{array}\right) & \text { if } x_{(0,0)}=0 .
\end{aligned}\right.
\end{aligned}
$$

It is easy to check that the cocycle (4.1) is not cohomologous to a homomorphism.

Since the group $G$ has normal subgroups of finite index the cocycle $c$ in (4.1) can be used to construct finite group extensions of the sft $X$. I have no example of a nontrivial cocycle $c: \mathbb{Z}^{d} \times X \longmapsto G$, where $G$ is abelian.

4.3. Colouring an Infinite Chessboard with Three Colours. Let $d=$ $2, A=\{0,1, \ldots, n-1\}, n \geq 3$,

$$
F=\left\{\begin{array}{l}
(0,1) \\
(0,0)(1,0)
\end{array}\right\}, \quad P=\left\{\left\{\begin{array}{c}
\text { not } j \\
j
\end{array} \text { not } j\right\}: j=0, \ldots, n-1\right\},
$$

and let $X^{(n)}=X_{(F, P)}$. The sft $X^{(n)}$ corresponds to all colourings of the lattice $\mathbb{Z}^{2}$ with $n$ colours so that no two horizontally or vertically adjacent lattice points have the same colour or, equivalently, to all colourings of an infinite chessboard with $n$ colours in which adjacent squares are coloured differently ([Lie], [Bax], [Sch2]).

Here we consider the case where $n=3$ and $X=X^{(3)}$. The equivalence relation $\Delta_{X}$ is topologically transitive, but not minimal: indeed, the periodic point

$$
\begin{aligned}
& .201201201 \text {. } \\
& \begin{array}{llllllll}
1 & 2 & 0 & 12 & 0 & 1 & 2 & 0
\end{array} \\
& \bar{y}=\begin{array}{lllllllllll}
. & 1 & 2 & 0 & 1 & 2 & 0 & 1 & 2 & 0 \\
. & 1 & 2 & 0 & 1 & 2 & 0 & 1 & 2 \\
. & 0 & 1 & 2 & 0 & 1 & 2 & 0 & 1
\end{array} ., \\
& \begin{array}{lllllllll}
12 & 0 & 1 & 2 & 0 & 1 & 2 & 0
\end{array} \text {. }
\end{aligned}
$$

satisfies that $\Delta_{X}(\bar{y})=\{\bar{y}\}$, whereas the equivalence class $\Delta_{X}(\bar{x})$ of the periodic point

$$
\bar{x}=\begin{array}{lllllllll}
0 & 1 & 0 & 1 & 0 & 1 & 0 & 1 & 0 \\
1 & 0 & 1 & 0 & 1 & 0 & 1 & 0 & 1 \\
.0 & 1 & 0 & 1 & 0 & 1 & 0 & 1 & 0 \\
.1 & 0 & 1 & 0 & 1 & 0 & 1 & 0 & 1 \\
.0 & 1 & 0 & 1 & 0 & 1 & 0 & 1 & 0
\end{array} .
$$

is dense in $X$ (the $\mathbf{0}$-coordinates of $\bar{y}$ and $\bar{x}$ are underlined). This can be verified by showing that every allowed colouring of an $n \times n$ square can be 
extended to an allowed colouring of a $(4 n-1) \times(4 n-1)$ square whose edges only carry the colours 0,1 . Consider, for example, the colouring

$$
\begin{array}{lll}
2 & 1 & 0 \\
1 & 0 & 1 \\
2 & 1 & 0
\end{array}
$$

of a $3 \times 3$ square. We extend this colouring to a $3 \times 6$ square by adding successively rows at the top which are coloured in the unique allowed colouring which is minimal in the lexicographic order:

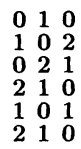

If the original square is $n \times n$ this method yields an extension of the colouring to an $2 n \times n$ rectangle whose top row only involves the colours 0,1 . Next we add $n$ columns on the left of the rectangle, which are again coloured in the lexicographically minimal way (going downwards), and observe that the left-most column only involves the colours 0,1 :

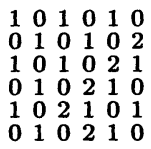

The final step is to reflect the $2 n \times 2 n$ colouring horizontally about the right-most column, and then vertically about the bottom row:

$\begin{array}{lllllllllll}1 & 0 & 1 & 0 & 1 & 0 & 1 & 0 & 1 & 0 & 1 \\ 0 & 1 & 0 & 1 & 0 & 2 & 0 & 1 & 0 & 1 & 0 \\ 1 & 0 & 1 & 0 & 2 & 1 & 2 & 0 & 1 & 0 & 1 \\ 0 & 1 & 0 & 2 & 1 & 0 & 1 & 2 & 0 & 1 & 0 \\ 1 & 0 & 2 & 1 & 0 & \frac{1}{1} & 0 & 1 & 2 & 0 & 1 \\ 0 & 1 & 0 & 2 & 1 & 0 & 1 & 2 & 0 & 1 & 0 \\ 1 & 0 & 2 & 1 & 0 & 1 & 0 & 1 & 2 & 0 & 1 \\ 0 & 1 & 0 & 2 & 1 & 0 & 1 & 2 & 0 & 1 & 0 \\ 1 & 0 & 1 & 0 & 2 & 1 & 2 & 0 & 1 & 0 & 1 \\ 0 & 1 & 0 & 1 & 0 & 2 & 0 & 1 & 0 & 1 & 0 \\ 1 & 0 & 1 & 0 & 1 & 0 & 1 & 0 & 1 & 0 & 1\end{array}$

The original coloured square is marked by underlining.

We claim that there exists, for every (nontrivial) locally compact, second countable, abelian group $G$, a Hölder cocycle $c: \mathbb{Z}^{2} \times X \longmapsto G$ which is not cohomologous to a homomorphism; this will imply in particular that $\Delta_{X}$ does not have $\mathbf{n}$-specification for any nonzero $\mathbf{n} \in \mathbb{Z}^{2}$. Indeed, let $a, b, c, \in G$, and 
let

$$
\begin{aligned}
& c((1,0), x)= \begin{cases}a & \text { if }\left(x_{(0,0)}, x_{(1,0)}\right)=(0,1), \\
-a & \text { if }\left(x_{(0,0)}, x_{(1,0)}\right)=(1,0), \\
b & \text { if }\left(x_{(0,0)}, x_{(1,0)}\right)=(0,2), \\
-b & \text { if }\left(x_{(0,0)}, x_{(1,0)}\right)=(2,0), \\
c & \text { if }\left(x_{(0,0)}, x_{(1,0)}\right)=(1,2), \\
-c & \text { if }\left(x_{(0,0)}, x_{(1,0)}\right)=(2,1),\end{cases} \\
& c((0,1), x)= \begin{cases}a & \text { if }\left(x_{(0,1)}, x_{(0,1)}\right)=(0,1), \\
-a & \text { if }\left(x_{(0,1)}, x_{(0,1)}\right)=(1,0), \\
b & \text { if }\left(x_{(0,1)}, x_{(0,1)}\right)=(0,2), \\
-b & \text { if }\left(x_{(0,1)}, x_{(0,1)}\right)=(2,0), \\
c & \text { if }\left(x_{(0,1)}, x_{(0,1)}\right)=(1,2), \\
-c & \text { if }\left(x_{(0,1)}, x_{(0,1)}\right)=(2,1) .\end{cases}
\end{aligned}
$$

A straightforward calculation shows that (4.2) determines a Hölder cocycle $c: \mathbb{Z}^{2} \times X \longmapsto G$, and that $c$ is not cohomologous to a homomorphism whenever $a+c \neq b$.

4.4. Colouring an Infinite Chessboard with $n \geq 4$ Colours. We begin with the case $n=4$ and define $X=X^{(4)}$ as in Example 4.3. If $m \geq 4$, and if $c=\left(c_{\mathbf{k}}, \mathbf{k} \in Q(m)\right)$ is an allowed colouring of $\partial Q(m)$ in the sense of Remark 2.3, where $Q(m)$ and $\partial Q(m)$ are defined as in the proof of Proposition 2.1, then we claim that there exists an allowed colouring $c^{\prime}=\left(c_{\mathbf{k}}^{\prime}, \mathbf{k} \in Q(m)\right)$ of $Q(m)$ such that $\pi_{\partial Q(m)}\left(c^{\prime}\right)=c$ (note that every allowed colouring of $Q(m)$ is of the form $\pi_{Q(m)}(x)$ for some $\left.x \in X\right)$.

In order to define the colouring $c^{\prime}$ we begin with the top left hand corner $(-m+1, m-1)$ of $Q(m-1)=Q(m) \backslash \partial Q(m)$. Since only two of the four neighbouring sites of $(-m+1, m-1)$ have been assigned colours, we can colour $(-m+1, m-1)$ different from these neighbours, and we assume for the sake of argument that we can colour $(-m+1, m-1)$ with an even colour (i.e. with a colour $i \in\{0,2\})$. Moving along the top edge of $Q(m-1)$ from left to right, we can colour the lattice sites alternately with odd and even colours without causing any clashes with the colours on $\partial Q(m)$, except for the last site $(m-1, m-1)$, where a different choice may be forced upon us. Let us call the corner $(m-1, m-1)$ good if $(m-1, m-1)$ has been assigned a colour with the correct parity (i.e. with a parity different form that of its neighbour to the left), and bad otherwise.

Starting with the site $(m-1, m-1)$ move down the right edge of the square $Q(m-1)$ by colouring the sites with alternating parity, bearing in 
mind the restrictions arising from the colours on $\partial Q(m)$. Again we can do this for all sites except possibly for $(m-1,-m+1)$, where we may be forced into a colour of the same parity as that of $(m-1,-m+2)$. Call the bottom right hand corder good or bad depending on whether $(m-1,-m+1)$ has a colour whose parity is different from that of $(m-1,-m+2)$ or not. With the same convention we continue along the bottom edge of $Q(m-1)$ from right to left, assign one of the labels good or bad to the bottom left hand corner of $Q(m-1)$, and then move up the left hand edge of $Q(m-1)$ with alternating parity, until we reach the site $(-m+1, m-2)$, where the parity of the available colour may be forced on us. The top left hand corner of $Q(m-1)$ is good if the parities of the colours on $(-m+1, m-3)$ and $(-m+1, m-1)$ are equal, and bad otherwise.

We claim that we may assume that at most two corners of $Q(m-1)$ are bad. Suppose for argument's sake that both bottom corners of $Q(m-1)$ are bad. Then we can colour the row $(-m+2,-m+2), \ldots,(m-2,-m+2)$ in such a manner that the parities of the colours on $(-m+1,-m+2), \ldots,(m-$ $1,-m+2)$ alternate. With the same definition of good and bad as before the bottom corners of the rectangle $\{-m+1, \ldots, m-1\} \times\{-m+2, \ldots, m-1\}$ are now both good (the fact that we may no longer be dealing with a square is irrelevant). Similarly one can deal with any other two adjacent bad corners of $Q(m-1)$, so that we are left with a rectangle $Q$ whose boundary $\partial Q$ is coloured with colours of alternating parity, except for breaks in the pattern in at most two bad corners, which must be diagonally opposite, and (possibly) in the top left hand corner, where we may have three adjacent coordinates coloured with the same parity. Starting from one of the good corners with strictly alternating parities we begin to colour the boundary $\partial Q^{\prime}$ of the rectangle $Q^{\prime}=Q \backslash \partial Q$ with colours of alternating parity as follows: if, for example, the top right hand corner of $Q^{\prime}$ is good, and if the parities of the colours there have the pattern

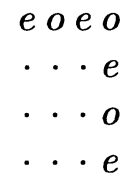

where ' $e$ ' is even and ' $o$ ' is odd, then we start colouring $\partial Q$ ' in the pattern

$$
\begin{aligned}
& \text { e o e } o \\
& \text { o e o e } \\
& \text { - e o } \\
& \text { - o e }
\end{aligned}
$$

A moment's thought shows that all of $\partial Q^{\prime}$ can be coloured with strictly 
alternating parity, and the interior of $Q^{\prime}$ can be filled in by colouring each site in a chessboard pattern with alternating even and odd colours.

From this argument it is clear that the equivalence relation $\Delta_{X}$ has $(1,0)$ specification. Since there exists a periodic point $\bar{x} \in X$ whose equivalence class $\Delta(\bar{x})$ is dense in $X$ we can apply Corollary 3.3 and conclude the triviality of all cocycles for the shift-action $\sigma$ of $\mathbb{Z}^{2}$ on $X^{(4)}$ with summable variation.

For $n>4$ the equivalence class $\Delta_{X^{(n)}}(\bar{x})$ is dense in $X^{(n)}$ for every periodic point $\bar{x} \in X^{(n)}$, and the $(1,0)$-specification of $\Delta(\bar{x})$ is obvious. Hence Corollary 3.3 applies, and all cocycles with summable variation on $X^{(n)}$ are trivial.

4.5. Square Ice and Related Shifts. Fix $n \geq 3$, and put

$$
\begin{aligned}
& B=\left\{\left[\begin{array}{c}
d^{c}{ }_{a}^{b} \\
\end{array}\right]: 1 \leq a, b, c, d \leq n-1 \text { and } a+b=c+d(\bmod n)\right\},
\end{aligned}
$$

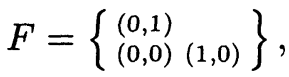

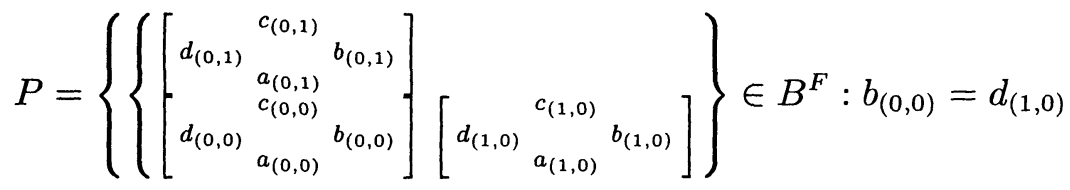

$$
\begin{aligned}
& \text { and } \left.c_{(0,0)}=a_{(0,1)}\right\} \text {, }
\end{aligned}
$$

and define $Y^{(n)}=X_{(F, P)}$ by (2.10). One can think of $B$ as a set of square tiles with coloured edges and of $Y^{(n)}$ as the set of all tilings of the plane by translates of these tiles such that touching edges of adjacent tiles are coloured identically, and one of the tiles has $(0,0)$ as a vertex.

Consider the map $\phi^{(n)}: X^{(n)} \longmapsto Y^{(n)}$ given by

$$
\phi^{(n)}(x)_{\mathbf{m}}=\left[\begin{array}{ll}
d_{\mathbf{m}} & a_{\mathbf{m}} \\
a_{\mathbf{m}} & b_{\mathbf{m}}
\end{array}\right]
$$

for every $x=\left(x_{\mathbf{n}}\right) \in X^{(n)}$ and $\mathbf{m}=\left(m_{1}, m_{2}\right) \in \mathbb{Z}^{2}$, where

$$
\begin{aligned}
& a_{\mathbf{m}}=x_{\left(m_{1}+1, m_{2}\right)}-x_{\left(m_{1}, m_{2}\right)} \quad(\bmod n), \\
& b_{\mathbf{m}}=x_{\left(m_{1}+1, m_{2}+1\right)}-x_{\left(m_{1}+1, m_{2}\right)} \quad(\bmod n), \\
& c_{\mathbf{m}}=x_{\left(m_{1}+1, m_{2}+1\right)}-x_{\left(m_{1}, m_{2}+1\right)} \quad(\bmod n), \\
& d_{\mathbf{m}}=x_{\left(m_{1}, m_{2}+1\right)}-x_{\left(m_{1}, m_{2}\right)} \quad(\bmod n) .
\end{aligned}
$$

It is easy to see that $\phi^{(n)}$ is continuous, surjective, and everywhere $n$-to- 1 . From the definition of $Y^{(n)}$ it is clear that the maps

$$
c((1,0), y)=a_{0} \quad(\bmod n), \quad c((0,1), y)=d_{0} \quad(\bmod n),
$$


for every

$$
y=\left(y_{\mathbf{n}}\right)=\left(\left[\begin{array}{cc}
d_{\mathbf{n}} & \\
d_{\mathbf{n}} & \\
& a_{\mathbf{n}}
\end{array}\right], \mathbf{n} \in \mathbb{Z}^{2}\right) \in Y^{(n)},
$$

define a Hölder cocycle $c=c^{(n)}: \mathbb{Z}^{2} \times Y^{(n)} \longmapsto \mathbb{Z} / n \mathbb{Z}$ which is not cohomologous to a homomorphism.

The sft $X^{(n)}$ is, in fact, a $\mathbb{Z} / n \mathbb{Z}$-extension of $Y^{(n)}$ : we define a homeomorphism $\eta: X^{(n)} \longmapsto X^{(n)}$ by setting $\eta(x)_{\mathbf{m}}=x_{\mathbf{m}}+1(\bmod n)$ for every $x=\left(x_{\mathbf{m}}\right) \in X^{(n)}$ and $\mathbf{m} \in \mathbb{Z}^{2}$, and observe that $\eta^{n}(x)=x$ and $\phi^{(n)}(x)=\phi^{(n)} \cdot \eta(x)$ for every $x \in X^{(n)}$. One can show that there exists a homeomorphism $\psi^{(n)}: X^{(n)} \longmapsto Y^{(n)} \times \mathbb{Z} / n \mathbb{Z}$ which carries the shift-action $\sigma$ of $\mathbb{Z}^{2}$ on $X^{(n)}$ to the skew-product action $\tau$ on $Y^{(n)} \times \mathbb{Z} / n \mathbb{Z}$ given by

$$
\tau_{\mathbf{n}}(y, k)=\left(\sigma_{\mathbf{n}}^{\prime}(y), k+c(\mathbf{n}, y)(\bmod n)\right.
$$

for every $\mathbf{n} \in \mathbb{Z}^{2}$ and $(y, k) \in Y \times \mathbb{Z} / n \mathbb{Z}$, where $\sigma^{\prime}$ is the shift-action of $\mathbb{Z}^{2}$ on $Y^{(n)}$.

Since the map $\phi^{(n)}: X^{(n)} \longmapsto Y^{(n)}$ is finite-to-one, and since $X^{(n)}$ has $d p p$ by Lemma 2.5 , the restriction of $\phi^{(n)}$ to $\Delta_{X^{(n)}}(x)$ must be injective for every $x \in X^{(n)}$, and a moment's thought reveals that $\phi^{(n)}$ actually induces a bijection of $\Delta_{X^{(n)}}(x)$ and $\Delta_{Y^{(n)}}\left(\phi^{(n)}(x)\right)$ for every $x \in X^{(n)}$.This shows that n-specification does not behave well under factor maps: since the shift-action of $\mathbb{Z}^{2}$ on $Y^{(n)}$ has a nontrivial continuous cocycle $c^{(n)}: \mathbb{Z}^{2} \times Y^{(n)} \longmapsto \mathbb{Z} / n \mathbb{Z}$ for every $n \geq 3$, none of the equivalence relations $\Delta_{Y(n)}$ can have $\mathbf{n}$-specification for any nonzero $\mathbf{n} \in \mathbb{Z}^{2}$. However, $\Delta_{X^{(n)}}$ has $(1,0)$-specification for every $n \geq 4$ by Example (4).

The sft $Y^{(3)}$ can be identified with a sft $Y$ investigated in [Bax] and [Lie], the so-called 'square ice' model. Suppose that any two horizontally or vertically adjacent lattice points in $\mathbb{Z}^{2}$ are linked by an arrow, and let $Y$ be the set of all configurations of such arrows with the property that every lattice point has two arrows pointing towards it, and two arrows pointing away from it. There are six possible patterns of arrows at any given lattice point in $\mathbb{Z}^{2}$ :

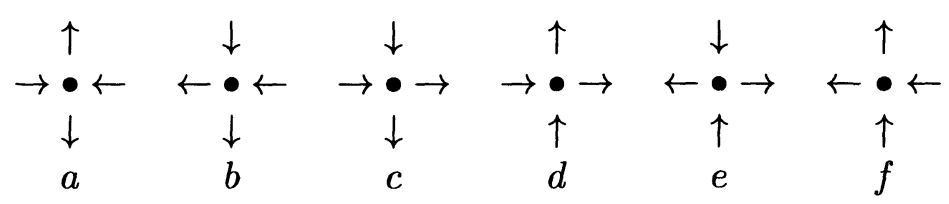

By labeling these patterns with the letters $a, b, c, d, e, f$, as indicated, we can regard $Y$ as the subshift $Y=X_{(F, P)} \subset\{a, b, c, d, e, f\}^{\mathbb{Z}^{2}}$ with

$$
F=\left\{\begin{array}{ll}
(0,1) \\
(0,0) & (1,0)
\end{array}\right\}
$$


and

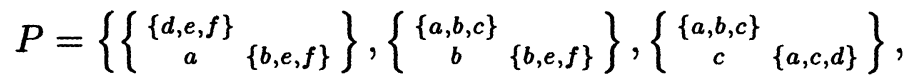

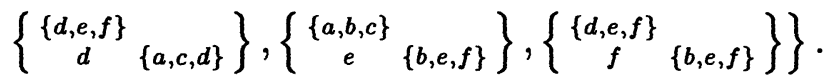

The notation used in the definition of $P$ in (4.4) should be interpreted as follows: if, for example, a point $y=\left(y_{\mathbf{n}}\right) \in Y$ satisfies that $y_{\mathbf{m}}=a$ for some $\mathbf{m}=\left(m_{1}, m_{2}\right) \in \mathbb{Z}^{2}$, then $y_{\left(m_{1}+1, m_{2}\right)} \in\{b, e, f\}$, and $y_{\left(m_{1}, m_{2}+1\right)} \in\{d, e, f\}$. The identification of $Y^{(3)}$ with $Y$ is obtained from the correspondence

$$
\begin{aligned}
& {\left[\begin{array}{cc}
2 & 2 \\
1 & 2 \\
1
\end{array}\right] \leftrightarrow a, \quad\left[\begin{array}{ll}
2 & 2 \\
2 & 2
\end{array}\right] \leftrightarrow b, \quad\left[\begin{array}{cc}
1 & 1 \\
1 & 1
\end{array}\right] \leftrightarrow c,} \\
& {\left[\begin{array}{ll}
1 & 2 \\
1 & 1
\end{array}\right] \leftrightarrow d, \quad\left[\begin{array}{cc}
1 & 1 \\
2 & 1
\end{array}\right] \leftrightarrow e, \quad\left[\begin{array}{l}
2 \\
2 \\
2
\end{array}\right]+\leftrightarrow f}
\end{aligned}
$$

of the alphabets $Y^{(3)}$ and $Y$.

By modifying the cocycle $c$ in (4.3) we can define a nontrivial Hölder cocycle $c^{\prime}: \mathbb{Z}^{2} \times Y^{(3)} \longmapsto \mathbb{Z}$ of the form

$$
\begin{aligned}
& c^{\prime}((1,0), y)= \begin{cases}1 & \text { if } a_{0}=1(\bmod 3), \\
-1 & \text { if } a_{0}=2(\bmod 3),\end{cases} \\
& c^{\prime}((0,1), y)= \begin{cases}1 & \text { if } d_{0}=1(\bmod 3), \\
-1 & \text { if } d_{0}=2(\bmod 3),\end{cases}
\end{aligned}
$$

for every

$$
y=\left(y_{\mathbf{n}}\right)=\left(\left[\begin{array}{cc}
d_{\mathbf{n}} & \\
d_{\mathbf{n}} & \\
a_{\mathbf{n}} & b_{\mathbf{n}}
\end{array}\right], \mathbf{n} \in \mathbb{Z}^{2}\right) \in Y^{(3)}
$$

However, since every integer-valued Hölder cocycle on $X^{(n)}$ is trivial for $n \geq 4$ (Example 4.4), the shift-action $\sigma$ on $Y^{(n)}$ does not admit a nontrivial Hölder cocycle $c^{\prime \prime}: \mathbb{Z}^{2} \times Y^{(n)} \longmapsto \mathbb{Z}$ if $n \geq 4$.

4.6. Lozenge Tilings. Consider the set $\Lambda$ of all tilings of the plane by tiles of the form
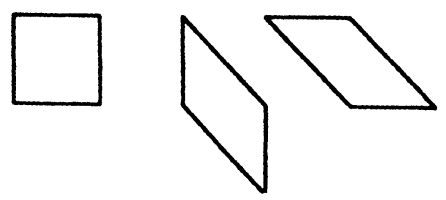

where the horizontal and vertical edges of the tiles have unit length, and the vertices of the tiles are located at in $\mathbb{Z}^{2} \subset \mathbb{R}^{2}$ (these tilings are isomorphic to the lozenge tilings discussed in [Thu]). Here is a typical partial tiling of 
the plane by lozenges:

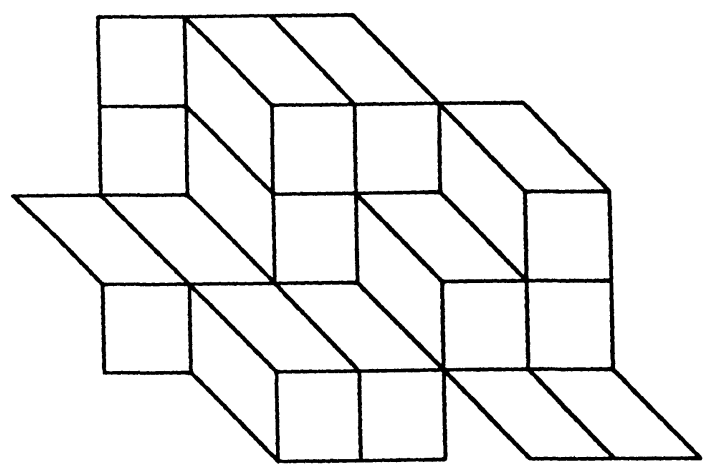

The set $\Lambda$ of all lozenge tilings is invariant under the shift-action $\tau$ of $\mathbb{Z}^{2}$ on $\mathbb{R}^{2}$. Each lozenge tiling corresponds to a unique tiling of the plane by the square tiles of unit size
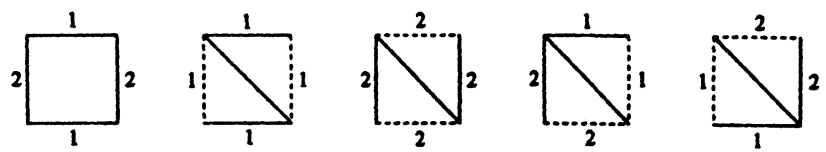

where the numbers attached to touching edges of adjacent tiles match, and the vertices of the tiles are located in $\mathbb{Z}^{2} \subset \mathbb{R}^{2}$. The correspondence between the two kinds of tilings is obtained by observing that the pattern of solid black lines arising from a tiling with the tiles in (4.8) determines a lozenge tiling and vice versa. A comparison of the tiles in (4.8) with those in (4.5) shows that $\Lambda$ may be identified with the closed, shift-invariant subset $Z \subset$ $Y^{(3)}$ consisting of all points which do not contain the symbol $\left[\begin{array}{ll}2 & 1 \\ 1 & 1\end{array}\right]$. In particular, the restriction to $Z$ of the cocycle $c^{\prime}: \mathbb{Z}^{2} \times Y^{(3)} \longmapsto \mathbb{Z}$ in (4.6) is a nontrivial Hölder cocycle for the shift-action of $\mathbb{Z}^{2}$ on $Z$ or, equivalently, for the $\mathbb{Z}^{2}$-action $\tau$ on $\Lambda$.

4.7. The Golden Mean. Let $d \geq 2$, and let $X \subset\{0,1\}^{\mathbf{z}^{d}}$ be the sft defined by the condition that no two adjacent coordinates of any point $x \in X$ are both equal to 1 (in other words, if $x_{\mathbf{n}}=1$ for some $x=\left(x_{\mathbf{n}}\right) \in X$ and $\mathbf{n} \in \mathbb{Z}^{d}$, then $x_{\mathbf{n} \pm \mathrm{e}^{(i)}}=0$ for $i=1, \ldots, d$, where $\mathrm{e}^{(i)}$ is the $i$-th unit vector in $\left.\mathbb{Z}^{d}\right)$. It is clear that $\Delta_{X}$ is minimal, and that $\Delta_{X}$ has $(\mathbf{n}, \xi)$-specification for every nonzero $\mathbf{n} \in \mathbb{Z}^{d}$ and $\xi \in(0,1)$. We conclude that Corollary 3.3 holds for this example. 


\section{Examples with zero entropy.}

The following example of a zero entropy $s f t X$ with a nontrivial Hölder cocycle $c: \mathbb{Z}^{2} \times X \longmapsto \mathbb{R}$ is due to W. Parry.

Example 5.1. Consider the two transition matrices

$$
H=\left(\begin{array}{lll}
1 & 1 & 0 \\
0 & 0 & 1 \\
1 & 1 & 0
\end{array}\right), \quad V=\left(\begin{array}{lll}
0 & 1 & 1 \\
1 & 0 & 0 \\
1 & 1 & 0
\end{array}\right),
$$

and define $X \subset\{0,1,2\}^{\mathbb{Z}^{2}}$ by the condition that an element $x=\left(x_{\mathbf{n}}\right) \in$ $\{0,1,2\}^{\mathbb{Z}^{2}}$ lies in $X$ if and only if $H\left(x_{\mathbf{n}}, x_{\mathbf{n}+(1,0)}\right)=$ $V\left(x_{\mathbf{n}}, x_{\mathbf{n}+(0,1)}\right)=1$ for every $\mathbf{n} \in \mathbb{Z}^{2}$. Put

$$
\begin{aligned}
& f_{1}(x)= \begin{cases}a & \text { if }\left(x_{(0,0)}, x_{(1,0)}\right) \in\{(0,0),(0,1)\}, \\
2 a-b & \text { if }\left(x_{(0,0)}, x_{(1,0)}\right)=(2,0), \\
2 a & \text { if }\left(x_{(0,0)}, x_{(1,0)}\right)=(2,1), \\
0 & \text { if }\left(x_{(0,0)}, x_{(1,0)}\right)=(1,2),\end{cases} \\
& f_{2}(x)= \begin{cases}a & \text { if }\left(x_{(0,0)}, x_{(0,1)}\right) \in\{(2,0),(2,1)\}, \\
b & \text { if }\left(x_{(0,0)}, x_{(0,1)}\right)=(0,1), \\
b-a & \text { if }\left(x_{(0,0)}, x_{(0,1)}\right)=(0,2), \\
0 & \text { if }\left(x_{(0,0)}, x_{(0,1)}\right)=(1,0),\end{cases}
\end{aligned}
$$

where $a, b \in \mathbb{R}$, and note that $f_{1} \cdot \sigma_{(0,1)}-f_{1}=f_{2} \cdot \sigma_{(1,0)}-f_{2}$. We define $c: \mathbb{Z}^{2} \times X \longmapsto \mathbb{R}$ by setting $c((1,0), \cdot)=f_{1}$ and $c((0,1), \cdot)=f_{2}$, and observe that, for a suitable choice of $a, b$, the cocycle $c$ is not cohomologous to a homomorphism. The basic idea underlying this example is that the matrix $H$ defines a one-dimensional $s f t Y$, and that $V$ induces a shift-commuting automorphism of $Y$ which preserves a Markov probability measure $\nu$ on $Y$ distinct from the measure of maximal entropy.

We continue with another set of examples which was discussed in [Led], [KS2], [KS3], [Sch1] and [KaS], where it was shown that every real-valued cocycle with summable variation on these shift spaces is continuously cohomologous to a homomorphism. In these examples the Gibbs relation $\Delta_{X}$ is trivial. In order to describe these examples we recall the terminology and notation of [Sch1] and [KS3]. Let $\mathfrak{R}_{d}=\mathbb{Z}\left[u_{1}^{ \pm 1}, \ldots, u_{d}^{ \pm 1}\right]$ be the ring of Laurent polynomials with integral coefficients in the commuting variables $u_{1}, \ldots, u_{d}$ and let $f \in \mathfrak{R}_{d}$. We write $f$ in the form $f=\sum_{\mathbf{m} \in \mathbb{Z}^{d}} c_{f}(\mathbf{m}) u^{\mathbf{m}}$ with $u^{\mathbf{m}}=u_{1}^{m_{1}} \cdots u_{d}^{m_{d}}$ and $c_{f}(\mathbf{m}) \in \mathbb{Z}$ for every $\mathbf{m}=\left(m_{1}, \ldots, m_{d}\right) \in \mathbb{Z}^{d}$, where $\sum_{\mathbf{m} \in \mathbb{Z}^{d}}\left|c_{f}(\mathbf{m})\right|<\infty$. If $\alpha$ is a $\mathbb{Z}^{d}$-action by automorphism of a compact, abelian group $X$, then the dual group $\mathfrak{M}=\hat{X}$ becomes a module over 
the ring $\mathfrak{R}_{d}$, where the action of $\mathfrak{R}_{d}$ on $\mathfrak{M}$ is defined by

$$
f \cdot a=\sum_{\mathbf{n} \in \mathbf{Z}^{d}} c_{f}(\mathbf{n}) \beta_{\mathbf{n}}(a)
$$

for all $a \in \mathfrak{M}$ and $f \in \mathfrak{R}_{d}$; here $\delta_{\mathbf{n}}=\widehat{\alpha_{\mathbf{n}}}$ is the automorphism of $\mathfrak{M}=\hat{X}$ duel to $\alpha_{\mathbf{n}}$. In particular

$$
\widehat{\alpha_{\mathbf{n}}}(a)=\beta_{\mathbf{n}}(a)=u^{\mathbf{n}} \cdot a
$$

for all $\mathbf{n} \in \mathbb{Z}^{d}$ and $a \in \mathfrak{M}$. Conversely, if $\mathfrak{M}$ is an $\mathfrak{R}_{d}$-module, and if

$$
\beta_{\mathbf{n}}^{\mathfrak{M}}(a)=u^{\mathbf{n}} \cdot a
$$

for every $\mathbf{n} \in \mathbb{Z}^{d}$ and $a \in \mathfrak{M}$, then we obtain a $\mathbb{Z}^{d}$-action

$$
\alpha^{\mathfrak{M}}: \mathbf{n} \mapsto \sigma_{\mathbf{n}}^{\mathfrak{M}}=\widehat{\beta_{\mathbf{n}}^{\mathfrak{N}}}
$$

on the compact, abelian group

$$
X^{\mathfrak{M}}=\widehat{\mathfrak{M}}
$$

dual to the $\mathbb{Z}^{d}$-action $\beta^{\mathfrak{M}}: \mathbf{n} \mapsto \beta_{\mathbf{n}}^{\mathfrak{N}}$ on $\mathfrak{M}$. From [KS1] and [Sch1] we know that $\mathfrak{M}$ is Noetherian whenever $\alpha^{\mathfrak{M}}$ is expansive, and duality theory implies that $X^{\mathfrak{M}}=\widehat{\mathfrak{M}}$ is zero-dimensional if and only if $\mathfrak{M}$ is a torsion group with respect to addition. Furthermore, if $X^{\mathfrak{M}}$ is zero-dimensional and $\sigma^{\mathfrak{M}}$ expansive, then there exists a finite, abelian group $G$ and a continuous, injective group homomorphism $\phi: X^{\mathfrak{M}} \longmapsto G^{\mathbb{Z}^{d}}$ such that $\phi \cdot \sigma_{\mathbf{n}}^{\mathfrak{M}}=\sigma_{\mathbf{n}} \cdot \phi$ for every $\mathbf{n} \in \mathbb{Z}^{d}$, where $\sigma$ is the shift-action of $\mathbb{Z}^{d}$ on $G^{\mathbb{Z}^{d}}$, and $Y=\phi(x) \subset G^{\mathbf{Z}^{d}}$ is a sft. The dynamical properties of $\sigma^{\mathfrak{M}}$ (like ergodicity and mixing with respect to the Haar measure on $X^{\mathfrak{M}}$ ) are discussed in [Sch1]; in particular, $\sigma^{\mathfrak{M}}$ is mixing if and only if multiplication by $\left(u^{\mathbf{n}}-1\right)$ on $\mathfrak{M}$ is injective for every nonzero element $\mathbf{n} \in \mathbb{Z}^{d}$.

Proposition 5.2. Let $\alpha$ be an expansive and mixing $\mathbb{Z}^{2}$-action on a compact, zero-dimensional, abelian group $X$, and let $\mathfrak{M}=\hat{X}$ be the $\mathfrak{R}_{2}$-module defined by (5.1)-(5.2). Suppose that $\left(u_{1}-1\right) \cdot \mathfrak{M} \cap\left(u_{2}-1\right) \cdot \mathfrak{M} \neq\left(u_{1}-1\right)\left(u_{2}-1\right) \cdot \mathfrak{M}$. Then there exists a finite, abelian group $G \subset \mathbb{S}$ and a Hölder cocycle $c$ : $\mathbb{Z}^{2} \times X \longmapsto G$, which is not cohomologous to a homomorphism, and which satisfies that $c(\mathbf{m}, \cdot): X \longmapsto G$ is a continuous group homomorphism for every $\mathbf{m} \in \mathbb{Z}^{2}$.

Proof. Let $\left.a \in\left(\left(u_{1}-1\right) \cdot \mathfrak{M} \cap\left(u_{2}-1\right) \cdot \mathfrak{M}\right)\right) \backslash\left(u_{1}-1\right)\left(u_{2}-1\right) \cdot \mathfrak{M}$. Then $a=\left(u_{2}-1\right) \cdot a_{1}=\left(u_{1}-1\right) \cdot a_{2}$ for some $a_{1}, a_{2} \in \mathfrak{M}$, but $a_{1}$ is not of the 
form $\left(u_{1}-1\right) \cdot b_{1}$ with $b_{1} \in \mathfrak{M}$. Since $a_{i} \in \mathfrak{M}=\hat{X}, a_{i}$ may be regarded as a homomorphism from $X$ into $\mathbb{T} \cong \mathbb{S}=\{z \in \mathbb{C}:|z|=1\}$, and it is not difficult to verify that this homomorphism sends $X$ to a finite subgroup of $\mathbb{T}$ and is Hölder. Since $a=\left(u_{2}-1\right) \cdot a_{1}=\left(u_{1}-1\right) \cdot a_{2}$, we can define a cocycle $c: \mathbb{Z}^{2} \times X \longmapsto \mathbb{T}$ by setting $c((1,0), \cdot)=a_{1}$ and $c((0,1), \cdot)=a_{2}$, and we claim that $c$ is not cohomologous to a homomorphism. Indeed, if $c$ were cohomologous to a homomorphism, then there would exist a Borel map $b: X \longmapsto \mathbb{T}$ and a constant $t \in \mathbb{T}$ such that $a_{1}=b \cdot \sigma_{(1,0)}-b+t$. Since multiplication by $u_{1}-1$ is injective on $\mathfrak{M}$, there exists a smallest $\mathfrak{R}_{2}$-module $\mathfrak{N} \supset \mathfrak{M}$ such that the multiplication by $u_{1}-1$ is invertible on $\mathfrak{N}$, and we set $\bar{b}=\left(u_{1}-1\right)^{-1} \cdot a_{1} \in \mathfrak{N}$. Put $Y=\hat{\mathfrak{N}}$ and write $\psi: Y \longmapsto X$ for the surjective group homomorphism dual to the inclusion $\mathfrak{M} \subset \mathfrak{N}$. According to [Sch1], $\alpha_{(1,0)}^{\mathfrak{N}}$ is ergodic, and our choice of $\bar{b}$ implies that $a_{1} \cdot \psi=\bar{b} \cdot \alpha_{(1,0)}^{\mathfrak{N}}-\bar{b}=$ $b \cdot \alpha_{(1,0)}^{\mathfrak{R}} \cdot \psi-b \cdot \psi+t=b \cdot \alpha_{(1,0)}^{\mathfrak{N}} \cdot \psi-b \cdot \psi+t$, so that $b \cdot \psi$ differs from $\bar{b}$ by a constant. Hence, $\bar{b}$ defines a character of $X$ and is thus an element of $\mathfrak{M}$, contrary to our assumption.

In order to test whether specific examples satisfy the condition of Proposition 5.2 it will be convenient to consider, for every $d \geq 1$ and every rational prime $p>1$, the ring $\mathfrak{R}_{d}^{(p)}=\mathbb{F}_{p}\left[u_{1}^{ \pm 1}, \ldots, u_{d}^{ \pm 1}\right]$ of Laurent polynomials in $u_{1}, \ldots, u_{d}$ with coefficients in the prime field $\mathbb{F}_{p}=\mathbb{Z} / p \mathbb{Z}$, and to denote by $h_{/ p} \in \mathfrak{R}_{d}^{(p)}$ the Laurent polynomial obtained by reducing the coefficients of a Laurent polynomial $h \in \mathfrak{R}_{d}$ modulo $p$. Since $\mathfrak{R}_{d}^{(p)}$ is a quotient ring of $\mathfrak{R}_{d}$, every $\mathfrak{R}_{d}^{(p)}$-module is also an $\mathfrak{R}_{d}$-module; in particular, if $h_{1}, \ldots, h_{k} \in \mathfrak{R}_{d}^{(p)}$, and if $\mathfrak{I}=\left(h_{1}, \ldots, h_{k}\right)=h_{1} \mathfrak{R}_{d}^{(p)}+\cdots+h_{k} \mathfrak{R}_{d}^{(p)} \subset \mathfrak{R}_{d}^{(p)}$, then the $\mathfrak{R}_{d}^{(p)}$ module $\mathfrak{M}=\mathfrak{R}_{d}^{(p)} / \mathfrak{I}$-regarded as an $\mathfrak{R}_{d}$-module-is equal to $\mathfrak{R}_{d} / \mathfrak{J}$, where $\mathfrak{J}=\left(p, f_{1}, \ldots, f_{k}\right)=p \mathfrak{R}_{d}+f_{1} \mathfrak{R}_{d}+\cdots+f_{k} \mathfrak{R}_{d}$, and where the $f_{1} \in \mathfrak{R}_{d}$ satisfy that $\left(f_{i}\right)_{/ p}=h_{i}$. As in $[\mathbf{K S 1}]$ and $[\mathbf{K S 2}]$ we may realize the $\mathbb{Z}^{d}$-action $\alpha^{\mathfrak{M}}$ as the shift-action of $\mathbb{Z}^{d}$ on the closed, shift-invariant subgroup

$$
X^{\mathfrak{M}}=\left\{x=\left(x_{\mathbf{m}}\right) \in(\mathbb{Z} / p \mathbb{Z})^{\mathbb{Z}^{d}}: \sum_{\mathbf{n} \in \mathbb{Z}^{d}} c_{f_{j}}(\mathbf{n}) x_{\mathbf{m}+\mathbf{n}}=0 \quad(\bmod p)\right.
$$

$$
\text { for every } \left.j=1, \ldots, k \text { and } \mathbf{m} \in \mathbb{Z}^{d}\right\} \text {. }
$$

Examples 5.3.(1) Let $p>1$ be a rational prime, $f \in \mathfrak{R}_{2}^{(p)}$ a polynomial with $f(1,1)=0 \quad(\bmod p), \mathfrak{I}=(f)=f \mathfrak{R}_{2}^{(2)}, \mathfrak{M}=\mathfrak{R}_{2}^{(2)} / \mathfrak{I}$, and assume that $\alpha^{\mathfrak{M}}$ is mixing. We claim that $\mathfrak{M}$ (regarded either as an $\mathfrak{R}_{2}$-module or as an $\mathfrak{R}_{2}^{(p)}$-module) satisfies the condition of Proposition 5.2.

The ideal $\mathfrak{m}=\left(u_{1}-1, u_{2}-1\right)=\left(u_{1}-1\right) \mathfrak{R}_{2}^{(p)}+\left(u_{2}-1\right) \mathfrak{R}_{2}^{(p)}$ is maximal in $\mathfrak{R}_{2}^{(p)}$, and consists of all Laurent polynomials $h \in \mathfrak{R}_{2}^{(p)}$ with $h(1,1)=$ 
$0 \quad(\bmod p)$. Hence there exist elements $f_{1}, f_{2} \in \mathfrak{R}_{2}^{(p)}$ with $\left(u_{1}-1\right) f_{1}+\left(u_{2}-\right.$ 1) $f_{2}=f$. Since $\alpha^{\mathfrak{M}}$ is mixing, $f$ is neither divisible by $u_{1}-1$ nor by $u_{2}-1$, and we conclude that $f_{1}$ is not divisible by $u_{2}-1$. We set $a_{i}=\left(u_{i}-1\right) f_{i}+\mathfrak{I}$ and conclude that $a_{1} \in\left(u_{1}-1\right) \cdot \mathfrak{M} \cap\left(u_{2}-1\right) \cdot \mathfrak{M}$, but $a_{1} \notin\left(u_{1}-1\right)\left(u_{2}-1\right) \cdot \mathfrak{M}$, which obviously implies that $\left(\left(u_{1}-1\right) \cdot \mathfrak{M} \cap\left(u_{2}-1\right) \cdot \mathfrak{M}\right) \backslash\left(u_{1}-1\right)\left(u_{2}-1\right) \cdot \mathfrak{M} \notin \emptyset$.

From Proposition 5.2 we conclude that there exists a nontrivial cocycle $c: \mathbb{Z}^{2} \times X^{\mathfrak{M}} \longmapsto \mathbb{T}$ such that $c((1,0), \cdot)=-a_{2}$ and $c((0,1), \cdot)=a_{1}$, where $a_{i}$ is regarded as a character of $X^{\mathfrak{M}}=\widehat{\mathfrak{M}}$.

In order to illustrate (1) we set $A=\{0,1\}=\mathbb{Z} / 2 \mathbb{Z}$, choose a nonempty, finite set $F \subset \mathbb{Z}^{d}$, and let

$$
\begin{aligned}
X(F)=\{x & =\left(x_{\mathbf{m}}\right) \in A^{\mathbb{Z}^{d}}: \sum_{\mathbf{m} \in F} x_{\mathbf{m}+\mathbf{n}} \\
& \left.=0 \quad(\bmod 2) \text { for every } \mathbf{n} \in \mathbb{Z}^{d}\right\} .
\end{aligned}
$$

Then $X(F)$ is a sft, and $X(F)=X^{\mathfrak{R}_{d}^{(2)} /(h)}$, where $h \in \mathfrak{R}_{d}^{(2)}$ is the Laurent polynomial $\sum_{\mathbf{n} \in F} u^{\mathbf{n}}$.

(2) Let $p=d=2$, and consider the polynomial $f=1+u_{1}+u_{2}+u_{2}^{2} \in \mathfrak{R}_{2}^{(2)}$ corresponding to the set $F=\{(0,0),(1,0),(0,1),(0,2)\} \subset \mathbb{Z}^{2}$ (cf. (5.6)). Then $f=\left(u_{1}-1\right) 1+\left(u_{2}-1\right) u_{2}$. If $\mathfrak{M}=\mathfrak{R}_{2}^{(2)} /(f)$ then we obtain, in the notation of $(5.6)$, that $c((1,0), x)=x_{(0,1)}$ and $c((0,1), x)=x_{(0,0)}$ for every $x=\left(x_{\mathbf{n}}\right) \in X^{\mathfrak{M}} \subset(\mathbb{Z} / 2 \mathbb{Z})^{\mathbb{Z}^{2}}$.

(3) Let $f=1+u_{1}+u_{2} \in \mathfrak{R}_{2}^{(3)}$ and $\mathfrak{M}=\mathfrak{R}_{2}^{(3)} /(f)$. Then $f=\left(u_{1}-1\right)+\left(u_{2}-\right.$ 1 ), and (1), Proposition 5.2, and (5.6) yield the cocycle $c: \mathbb{Z}^{2} \times X \longmapsto \mathbb{Z} / 3 \mathbb{Z}$ given by $c((1,0), x)=2 x_{(0,0)}$ and $c((0,1), x)=x_{(0,0)}$ for every $x=\left(x_{\mathbf{n}}\right) \in$ $X^{\mathfrak{M}} \subset(\mathbb{Z} / 3 \mathbb{Z})^{\mathbb{Z}^{2}}$.

(4) Let $f=1+u_{1}+u_{1}^{2}+u_{2}+u_{1} u_{2}+u_{2}^{2} \in \mathfrak{R}_{2}^{(2)}$ and $\mathfrak{M}=\mathfrak{R}_{2}^{(2)} /(f)$. Then $f=\left(u_{1}-1\right)\left(u_{1}+u_{2}\right)+\left(u_{2}-1\right)^{2}$, and the cocycle $c: \mathbb{Z}^{2} \times X \longmapsto \mathbb{Z} / 2 \mathbb{Z}$ is given by $c((1,0), x)=x_{(0,0)}+x_{(0,1)} \quad(\bmod 2)$ and $c((0,1), x)=x_{(1,0)}+x_{(0,1)}$ $(\bmod 2)$ for every $x=\left(x_{\mathbf{n}}\right) \in X^{\mathfrak{M}} \subset(\mathbb{Z} / 2 \mathbb{Z})^{\mathbb{Z}^{2}}$.

(5) Let $f=1+u_{1}+u_{2}+u_{2}^{2}+u_{2}^{3}+u_{2}^{4} \in \mathfrak{R}_{2}^{(2)}$ and $\mathfrak{M}=\mathfrak{R}_{2}^{(2)} /(f)$. Since $f=\left(u_{1}-1\right)+\left(u_{2}-1\right)\left(u_{2}+u_{2}^{3}\right)$ we obtain the cocycle $c: \mathbb{Z}^{2} \times X \longmapsto \mathbb{Z} / 2 \mathbb{Z}$ with $c((1,0), x)=x_{(0,1)}+x_{(0,3)}(\bmod 2)$ and $c((0,1), x)=x_{(0,0)}$ for every $x=\left(x_{\mathbf{n}}\right) \in X^{\mathfrak{M}} \subset(\mathbb{Z} / 2 \mathbb{Z})^{\mathbb{Z}^{2}}$.

(6) Let $f=u_{1}^{4}+u_{2}\left(u_{1}^{2}+u_{1}^{3}\right)+u_{2}^{2}\left(1+u_{1}+u_{1}^{2}\right)+u_{2}^{3}+u_{2}^{4} \in \mathfrak{R}_{2}^{(2)}$ and $\mathfrak{M}=\mathfrak{R}_{2}^{(2)} /(f)$. Then $f=\left(u_{1}-1\right)\left(1+u_{1}+u_{1}^{2}+u_{1}^{3}+u_{1}^{2} u_{2}+u_{1} u_{2}^{2}\right)+\left(u_{2}-\right.$ 1) $\left(1+u_{2}+u_{2}^{3}\right)$, and we obtain the cocycle $c: \mathbb{Z}^{2} \times X \longmapsto \mathbb{Z} / 2 \mathbb{Z}$ with $c((1,0), x)=x_{(0,0)}+x_{(0,1)}+x_{(0,3)}(\bmod 2)$ and $c((0,1), x)=x_{(0,0)}+x_{(1,0)}+$ $x_{(2,0)}+x_{(3,0)}+x_{(2,1)}+x_{(1,2)}(\bmod 2)$ for every $x=\left(x_{\mathbf{n}}\right) \in X^{\mathfrak{M}} \subset(\mathbb{Z} / 2 \mathbb{Z})^{\mathbb{Z}^{2}}$. 
(7) Let $p>1, d \geq 2$, and let $f \in \mathfrak{R}_{d}^{(p)}$ be a Laurent polynomial with $f(1, \ldots, 1)=0(\bmod p)$ such that $\alpha^{\mathfrak{M}}$ is mixing, where $\mathfrak{M}=\mathfrak{R}_{d}^{(p)} /(f)$. From (5.6) we see that $\alpha=\alpha^{\mathfrak{M}}$ has $p$ fixed points, and we write $G=F i x\left(\alpha^{\mathfrak{M}}\right) \cong$ $\mathbb{Z} / p \mathbb{Z}$ for the group of fixed points of $\alpha$. Since $G \subset X=X^{\mathfrak{M}}$ is a closed, $\alpha$-invariant subgroup, we can consider the quotient group $Y=X / G$ and write $\alpha^{\prime}$ for the $\mathbb{Z}^{d}$-action induced by $\alpha$ on $Y$. Elementary duality theory shows that the $\mathfrak{R}_{d}$-module arising from $\alpha^{\prime}$ via (5.3)-(5.5) is of the form $\mathfrak{N}=\left(u_{1}-1\right) \cdot \mathfrak{M}+\cdots+\left(u_{d}-1\right) \cdot \mathfrak{M} \subset \mathfrak{M}$. If the modules $\mathfrak{M}$ and $\mathfrak{N}$ are isomorphic then the $\mathbb{Z}^{d}$-actions $\alpha \mathfrak{M}$ and $\alpha^{\prime}$ are topologically and algebraically conjugate, and we obtain a $p$-to-1 surjective group homomorphism $\phi^{\mathfrak{M}} \longmapsto$ $X^{\mathfrak{M}}$ which commutes with the $\mathbb{Z}^{d}$-action and $\alpha$ and therefore (by general nonsense about group-extensions of dynamical systems) a nontrivial cocycle $c: \mathbb{Z}^{d} \times X \longmapsto \mathbb{Z} / p \mathbb{Z}$. The (obvious) necessary and sufficient condition for $\mathfrak{M}$ and $\mathfrak{N}$ to be isomorphic as $\mathfrak{R}_{d}$-modules (or, equivalently, as $\mathfrak{R}_{d}^{(p)}$ modules) is that $\mathfrak{N}$ is cyclic, i.e. that there exists an element $b \in \mathfrak{N}$ such that $\mathfrak{N}=\mathfrak{R}_{d} \cdot b=\mathfrak{R}_{d}^{(p)} \cdot b$.

When is $\mathfrak{N}$ cyclic? The answer is sometimes yes and sometimes no. For example, if $d=p=2$ and $f=1+u_{1}+u_{2}+u_{2}^{2}$ as in Example (2), then $\left(u_{1}-1\right) \cdot \mathfrak{M}=\left(u_{2}-1\right) \cdot \mathfrak{M}$ and $\mathfrak{N}=\left(u_{1}-1\right) \cdot \mathfrak{M}$, so that $\mathfrak{N}$ is cyclic. Similarly, if $d=2, p=3$, and $f=1+u_{1}+u_{2}$ (Example $\left.(3)\right)$, then $\left(u_{1}-1\right) \cdot \mathfrak{M}=\left(u_{2}-1\right) \cdot \mathfrak{M}$, and $\mathfrak{N}=\left(u_{1}-1\right) \cdot \mathfrak{M}$ is cyclic. In Example (5) we see that $\left(u_{1}-1\right) \cdot \mathfrak{M} \subsetneq$ $\left(u_{2}-1\right) \cdot \mathfrak{M}$, so that $\mathfrak{N}=\left(u_{2}-1\right) \cdot \mathfrak{M}$ is again cyclic. In Example (6), $\left(u_{1} u_{2}^{-1}-1\right) \cdot \mathfrak{M}=\left(u_{1}-1\right) \cdot \mathfrak{M} \cap\left(u_{2}-1\right) \cdot \mathfrak{M}$, but $\left(u_{1} u_{2}^{-1}-1\right) \cdot \mathfrak{M} \subsetneq\left(u_{1}-1\right) \cdot \mathfrak{M}$ and $\left(u_{1} u_{2}^{-1}-1\right) \cdot \mathfrak{M} \subsetneq\left(u_{2}-1\right) \cdot \mathfrak{M}$. These conditions can be expressed dynamically: in the Examples (2) and (3) every point $x \in X=X^{\mathfrak{M}}$ which is fixed under $\alpha_{(1,0)}$ is also fixed under $\alpha_{(0,1)}$ and vice versa. In Example (5) every point which is fixed under $\alpha_{(0,1)}$ is fixed under $\alpha_{(1,0)}$, but not the other way round. In Example (6) there exist fixed points for $\alpha_{(1,0)}$ which are not fixed under $\alpha_{(0,1)}$, and vice versa, but every point which is fixed under $\alpha_{(1,-1)}$ is fixed under $\alpha_{(1,0)}$ and $\alpha_{(0,1)}$.

We conclude that the $\mathbb{Z}^{2}$-actions $\alpha^{\mathfrak{M}}$ appearing in Examples (2), (3), (5), and (6), admit continuous, surjective, everywhere two-to-one group homomorphisms $\phi: X^{\mathfrak{M}} \longmapsto X^{\mathfrak{M}}$ which commute with $\alpha^{\mathfrak{M}}$.

On the other hand, if $f=1+u_{1}+u_{1}^{2}+u_{2}+u_{1} u_{2}+u_{2}^{2} \in \mathfrak{R}_{2}^{(2)}$ and $\mathfrak{M}=\mathfrak{R}_{2}^{(2)} /(f)$ as in Example (4), then $\mathfrak{N}=\left(u_{1}-1\right) \cdot \mathfrak{M}+\left(u_{2}-1\right) \cdot \mathfrak{M}$ is not isomorphic to $\mathfrak{M}$, and hence not cyclic. In fact, if $G=F i x\left(a^{\mathfrak{M}}\right) \cong \mathbb{Z} / 2 \mathbb{Z}$ is the group of fixed points of $\alpha^{\mathfrak{M}}$ and $\alpha^{\prime}$ the action induced by $\alpha^{\mathfrak{M}}$ on $Y=X^{\mathfrak{M}} / G$, then the $\mathbb{Z}^{2}$-actions $\alpha^{\mathfrak{M}}$ and $\alpha^{\prime}$ are not topologically conjugate. In order to verify this we note that $\alpha^{\mathfrak{M}}$ has two fixed points (the elements of $G$ ), whereas $\alpha^{\prime}$ has 4 fixed points in $Y$ : they are the elements of $\left(G_{(1,0)}+G_{(0,1)}\right) / G$, where $G_{(1,0)}$ and $G_{(0,1)}$ are the points in $X^{\mathfrak{M}}$ which 
are invariant under $\alpha_{(1,0)}^{\mathfrak{M}}$ and $\alpha_{(0,1)}^{\mathfrak{M}}$, respectively. Hence the $\mathbb{Z}^{2}$-action $\alpha^{\prime}$ is not topologically conjugate to $\alpha^{\mathfrak{M}}$, and the modules $\mathfrak{M}$ and $\mathfrak{N}$ are not isomorphic ([Sch1] ).

So far we have only discussed examples with zero entropy whose Hölder cohomology is nontrivial. Here is a simple example with zero entropy and trivial Hölder cohomology.

Example 5.4. Let $d=3, p=2$, and let $F \subset \mathbb{Z}^{3}$ be the set

$$
\{(0,0,0),(1,0,0),(0,1,0),(0,0,1),(0,0,-1)\}
$$

corresponding to the Laurent polynomial $h=1+u_{1}+u_{2}+u_{3}+u_{3}^{-1} \epsilon$ $\mathfrak{R}_{3}^{(2)}$. The subshift $X=X(F)$ in (5.7) is topologically mixing and has zero entropy, and the Gibbs relation $\Delta_{X}$ is trivial. However, if $G$ is a locally compact, second countable group with a given doubly invariant metric, then every cocycle $c: \mathbb{Z}^{3} \times X \longmapsto G$ with summable variation is trivial. In order to verify this we put $S=\left\{\left(k_{1}, k_{2}, k_{3}\right) \in \mathbb{Z}^{3}: k_{3} \in\{0,1\}\right\}$ and observe that the coordinate projection $\sigma_{S}: X \longmapsto(\mathbb{Z} / 2 \mathbb{Z})^{S}$ is a continuous group isomorphism of $X$ and $Y=(\mathbb{Z} / 2 \mathbb{Z})^{S}$. We write $\tau$ for the obvious shift-action of $\mathbb{Z}^{2}$ on $Y$ and note that $\sigma_{S} \cdot \sigma_{\left(k_{1}, k_{2}, 0\right)}=\tau_{\left(k_{1}, k_{2}\right)} \cdot \pi_{S}$ for every $\left(k_{1}, k_{2}\right) \in \mathbb{Z}^{2}$, where $\sigma$ is the shift-action of $\mathbb{Z}^{2}$ on $X$. If $c: \mathbb{Z}^{3} \times X \longmapsto G$ is a cocycle for $\sigma$ with summable variation, we set, for every $\mathbf{k}=\left(k_{1}, k_{2}\right) \in \mathbb{Z}^{2}, c^{\prime}(\mathbf{k}, y)=$ $c\left(\left(k_{1}, k_{2}, 0\right), \pi_{S}^{-1}(y)\right)$, and obtain a cocycle $c^{\prime}: \mathbb{Z}^{2} \times Y$ for $\tau$ which again has summable variation; if $c$ is Hölder then $c^{\prime}$ is also Hölder. However, since $\tau$ is the shift-action of $\mathbb{Z}^{2}$ on a full shift space, Example 4.1 shows that $c^{\prime}$ must be (continuously or Hölder) cohomologous to a homomorphism, which implies the triviality of $c$.

Another interesting phenomenon occurs if $p=d=2$, and if $F \subset \mathbb{Z}^{2}$ is the set $\{(0,0),(1,0),(0,1)\}$ corresponding to the polynomial $h=1+u_{1}+u_{2} \subset$ $\mathfrak{R}_{2}^{(2)}$ (cf. (5.7)). We set $\mathfrak{M}=\mathfrak{R}_{2}^{(2)} /(h)$ and define $X(F)=X^{\mathfrak{M}}$ by (5.6) or $(5.7)$. Then $h(0,0) \neq 0(\bmod 2)$, so that we are not in the situation of Example $5.3(1)$. Indeed, since $\left(u_{1}-1\right)+(h)=u_{2}+(h)$ and $\left(u_{1}-1\right) \cdot \mathfrak{M}=$ $u_{2} \cdot \mathfrak{M}=\mathfrak{M}=\left(u_{2}-1\right) \cdot \mathfrak{M}=\left(u_{1}-1\right)\left(u_{2}-1\right) \cdot \mathfrak{M}$, the hypothesis of Proposition 5.2 is not satisfied, and there is no nontrivial cocycle $c: \mathbb{Z}^{2} \times X \longmapsto \mathbb{Z} / 2 \mathbb{Z}$ of the special form described in Proposition 5.2. One can actually prove much more.

Proposition 5.5. Let $p=d=2, h=1+u_{1}+u_{2} \in \mathfrak{R}_{2}^{(2)}, \mathfrak{M}=\mathfrak{R}_{2}^{(2)} /(h)$, and define the shift-action $\sigma=\alpha^{\mathfrak{M}}$ of $\mathbb{Z}^{2}$ on $X=X^{\mathfrak{M}} \subset\{0,1\}^{\mathbb{Z}^{2}}$ as in (5.6)(5.7). If $G$ is a countable, discrete, abelian group, then every continuous cocycle $c: \mathbb{Z}^{2}: X \longmapsto G$ for $\sigma$ is continuously cohomologous to a homomorphism. However, $\sigma$ does have nontrivial continuous cocycles with values in 
nonabelian, discrete groups.

Proof. Let $c: \mathbb{Z}^{2} \times X \longmapsto G$ be a cocycle with summable variation, and put $f=c((1,0), \cdot)$. In view of the cocycle equation (3.4) we may assume without loss in generality that $f$ only depends on the coordinates in $H=\left\{\left(m_{1}, m_{2}\right) \in \mathbb{Z}^{3}: m_{2} \geq 0\right\}$, and hence on the coordinates in $L=$ $\{(m, 0): m \in \mathbb{Z}\}$, since the coordinates $\left\{x_{\mathbf{m}}: \mathbf{m} \in H\right\}$ of a point $x \in X$ are completely determined by the coordinates $\left\{x_{\mathbf{m}}: \mathbf{m} \in L\right\}$. As $G$ is discrete, there exists an integer $m \geq 0$ such that $f$ is a function of the coordinates $(-m, 0), \ldots,(m, 0)$, and does not depend on the coordinates $x_{(k, 0)}$ with $|k|>m$. After subtracting a constant from $f$ (which amounts to modifying $c$ by a homomorphism) we may also assume that $f(0, \ldots, 0)=0$.

If $n \geq 0$, and if $a_{-n}, \ldots, a_{n}$ is an arbitrary sequence of 0 's and 1's, we consider an element $y \in X$ with

$$
y_{(k, 0)}= \begin{cases}a_{k} & \text { if } k \leq n \\ 0 & \text { if }|k|>n\end{cases}
$$

and set

$$
F(y)=\sum_{k \in \mathbb{Z}} f\left(\sigma_{(k, 0)}(y)\right) .
$$

Then $F(y)$ is well defined, and we claim that $F(y)=0$, irrespective of the choice of $n$ and $a_{-n}, \ldots, a_{n}$. Indeed, the cocycle equation (3.4) implies that $F(y)=F\left(\sigma_{(0, r)}(y)\right)$ for every $r \geq 0$, and by setting $r=2^{s}$ for sufficiently large $s \geq 1$ we see that $F(y)=2 F(y)=0$ (a computation shows that the sequence $\left(y_{\left(k, 2^{s}\right)}, k \in \mathbb{Z}\right)$ is of the form

$$
\ldots, 0,0, a_{-n}, \ldots, a_{n}, 0, \ldots, 0, a_{-n}, \ldots, a_{n}, 0,0, \ldots,
$$

where the gap between the two occurrences of the block $a_{-n}, \ldots, a_{n}$ is arbitrarily large for sufficiently large $s$ ).

By applying Proposition 3.1 to the function $f$ (regarded as a function on $\{0,1\}^{\mathbf{Z}}$ with summable variation) we conclude that there exists a continuous map $b:\{0,1\}^{\mathbb{Z}} \longmapsto G$ such that $f(x)=b\left(\sigma_{(1,0)}(x)\right)-b(x)$ for all $x \in\{0,1\}^{\mathbb{Z}}$, which implies that $f: X \longmapsto G$ is of the form $b^{\prime} \cdot \sigma_{(1,0)}-b^{\prime}$ for some continuous function $b^{\prime}: X \longmapsto G$. The ergodicity of $\sigma_{(1,0)}$ allows us to conclude that $c$ is continuously cohomologous to a homomorphism.

The last assertion is proved as follows. For every $n \geq 1$ the group $\Phi(2 n+$ $1)=\left\{x \in X: \sigma_{(2 n+1,0)}(x)=x\right\}$ is finite, nontrivial, and $\sigma$-invariant, and we put $H(2 n+1)=\left\{\mathbf{n} \in \mathbb{Z}^{2}: \sigma_{\mathbf{n}}(x)=x\right.$ for all $\left.x \in \Phi(2 n+1)\right\}$. Let $G(2 n+1)=\left(\mathbb{Z}^{2} / H(2 n+1)\right) \times \Phi(2 n+1)$, furnished with the group operation $(\mathbf{m}+H(2 n+1), x) \cdot\left(\mathbf{m}^{\prime}+H(2 n+1), x^{\prime}\right)=\left(\mathbf{m}+\mathbf{m}^{\prime}+H(2 n+1), x+\sigma_{\mathbf{m}}\left(x^{\prime}\right)\right)$. 
Then $G(2 n+1)$ is finite and nonabelian, and we may identify $\Phi(2 n+1)$ with the normal subgroup $\{(H(2 n+1), x): x \in \Phi(2 n+1)\}$ of $G(2 n+1)$.

We fix $n \geq 1$ for the moment, put $Y=X / \Phi(2 n+1)$, and let $\sigma^{\prime}$ be the $\mathbb{Z}^{2}$-action on $Y$ induced by $\sigma$. Since $\hat{Y}=\left(u_{1}^{2 n+1}-1\right) \cdot \mathfrak{M} \cong \mathfrak{M}$, there exists a continuous group isomorphism $\eta: Y \longmapsto X$ such that $\eta \cdot \sigma_{\mathbf{n}}^{\prime}=\sigma_{\mathbf{n}} \eta$ for every $\mathbf{n} \in \mathbb{Z}^{2}$. Define a homeomorphism $\psi: X \longmapsto Y \times \Phi(2 n+1)$ by $\psi(x)=(x+\Phi(2 n+1), \zeta(x))$, where $\zeta(x) \in \Phi(2 n+1)$ is the unique element satisfying that $x_{(i, 0)}=(\zeta(x))_{(i, 0)}$ for $i=0, \ldots, 2 n-1$. Since $\zeta(x)=\zeta\left(x^{\prime}\right)$ whenever $x+\Phi(2 n+1)=x^{\prime}+\Phi(2 n+1)$, we may regard $\zeta$ as a continuous map $\zeta: Y \longmapsto \Phi(2 n+1)$. Put $v(\mathbf{m}, y)=\zeta\left(\sigma_{\mathbf{m}}(y)\right)-\sigma_{(\mathbf{m})}(\zeta(y))$ for every $y \in Y$ and $\mathbf{m} \in \mathbb{Z}^{2}$ and observe that $\psi\left(\sigma_{\mathbf{m}}(x)\right)=\left(\sigma_{\mathbf{m}}(x)+\Phi(2 n+1), \sigma_{\mathbf{m}}(\zeta(x+\right.$ $\Phi(2 n+1)))+v(\mathbf{m}, x+\Phi(2 n+1)))$, for all $x \in X$ and $\mathbf{n} \in \mathbb{Z}^{2}$. The map $v: \mathbb{Z}^{2} \times Y \longmapsto \Phi(2 n+1)$ satisfies that $v(\mathbf{m}+\mathbf{n}, y)=v\left(\mathbf{m}, \sigma_{\mathbf{n}}^{\prime}(y)\right)+\sigma_{\mathbf{m}}(v(\mathbf{n}, y))$ for all $\mathbf{m}, \mathbf{n} \in \mathbb{Z}^{2}$ and $y \in Y$, and we obtain a cocycle $c_{2 n+1}: \mathbb{Z}^{2} \times X \longmapsto$ $G(2 n+1)$ by setting $c_{2 n+1}(\mathbf{m}, \eta(y))=(\mathbf{m}+H(2 n+1), v(\mathbf{m}, y))$ for all $\mathbf{m} \in \mathbb{Z}^{2}$ and $y \in Y$.

In order to prove that $c_{2 n+1}$ is not cohomologous to a homomorphism we define a skew-product action $\tau$ of $H(2 n+1)$ on $Y \times \Phi(2 n+1)$ as in (3.11) by $\tau_{\mathbf{m}}(y, x)=\left(\sigma_{\mathbf{m}}^{\prime}(y), v(\mathbf{m}, y)+x\right)$ for every $\mathbf{m} \in H(2 n+1), y \in Y$, and $x \in \Phi(2 n+1)$. Then $\tau_{\mathbf{m}} \cdot \psi=\psi \cdot \sigma_{\mathbf{m}}$ for every $\mathbf{m} \in H(2 n+1)$, which implies that $\tau$ is topologically mixing (cf. [Led]). A straightforward calculation shows that this implies the nontriviality of $c_{2 n+1}$ for every $n \geq 1$.

\section{References}

[Bax] R.J. Baxter, Exactly solved models in statistical mechanics, Academic Press, New York and London, 1982.

[FeM] J. Feldman and C.C. Moore, Ergodic equivalence relations, cohomology, and von Neumann algebras. I, Trans. Amer. Math. Soc., 234 (1977), 289-324.

[GePr] W. Geller and J. Propp, The fundamental group of a $\mathbb{Z}^{2}$-shift, preprint.

[Kam1] J.W. Kammeyer, A complete classification of two-point extensions of a multidimensional Bernoulli shift, J. Analyse Math., 54 (1990), 113-163.

[Kam2] _ A classification of the isometric extensions of a multidimensional Bernoulli shift, Ergod. Th. \& Dynam. Sys., 12 (1992), 267-282.

[Kas] P.W. Kasteleyn, The statistics of dimers on a lattice. I, Physica, 27 (1961), 12091225.

[KaS] A. Katok and K. Schmidt, The cohomology of expansive $\mathbb{Z}^{d}$-actions by automorphisms of compact, abelian groups, Pacific J. of Math., 170 (1995), 105-144.

[KaSp] A. Katok and R.J. Spatzier, Differential rigidity of hyperbolic abelian actions, preprint, 1992.

[KS1] B. Kitchens and K. Schmidt, Automorphisms of compact groups, Ergod. Th. \& Dynam. Sys., 9 (1989), 691-735. 
[KS2] - Markov subgroups of $\left(Z_{/ 2}\right)^{\mathbf{z}^{2}}$, Contemp. Math., Amer. Math. Soc., 135 (1992), 265-283.

[KS3] - Mixing sets and relative entropies for higher dimensional Markov shifts, Ergod. Th. \& Dynam. Sys., 13 (1993), 705-735.

[Led] F. Ledrappier, Un champ markovien peut être d'entropie nulle et mélangeant, C. R. Acad. Sci. Paris Ser. A, 287 (1978), 561-562.

[Lie] E.H. Lieb, Residual entropy of square ice, Phys. Rev., 162 (1967), 162-172.

[LSW] D. Lind, K. Schmidt, and T. Ward, Mahler measure and entropy for commuting automorphisms of compact groups, Invent. Math., 101 (1990), 593-629.

[Mic] E. Michael, Continuous selections II, Ann. of Math., 64 (1956), 562-580.

[Rue] D. Ruelle, Thermodynamic Formalism, Addison-Wesley, Reading, Mass., 1978.

[Sch1] K. Schmidt, Automorphisms of compact abelian groups and affine varieties, Proc., London Math. Soc., 61 (1990), 480-496.

[Sch2] _ Algebraic ideas in ergodic theory, CBMS Lecture Notes, Vol. 76, Amer. Math. Soc., 1990.

[TeL] H.N.V. Temperley and E.H. Lieb, Relations between the 'percolation' and 'colouring' problem and other graph-theoretical problems associated with regular planar lattices: some exact results for the 'percolation' problem, Proc. Roy. Soc. London Ser. A, 322 (1971), 251-280.

[Thu] W.P. Thurston, Groups, tilings, and finite state automata, Summer 1989 AMS Colloquium Lectures.

Received Decmeber 7, 1992. This investigation was sparked off by discussions with A. Katok during a visit to Pennsylvania State University in December 1991. The author would also like to thank W. Parry for permission to include Example 5.1, and to Jim Propp for useful discussions of domino tilings. Research at MSRI supported in part by NSF grant \#DMS 9022140.

MATHEMATICS INSTITUTE

UNIVERSITY OF VIENNA

VIENNA, A-1090

VIENNA, AUSTRIA

E-MAIL ADDRESS: KLAUS.SCHMIDT@UNIVIE.AC.AT 



\title{
PACIFIC JOURNAL OF MATHEMATICS
}

Founded by E. F. Beckenbach (1906-1982) and F. Wolf (1904-1989)

\section{EDITORS}

Sun-Yung Alice Chang (Managing Editor)

University of California

Los Angeles, CA 90095-1555

pacific@math.ucla.edu

F. Michael Christ

University of California

Los Angeles, CA 90095-1555

christ@math.ucla.edu

Thomas Enright

University of California

San Diego, La Jolla, CA 92093

tenright@ucsd.edu

Nicholas Ercolani

University of Arizona

Tucson, AZ 85721

ercolani@math.arizona.edu
Robert Finn

Stanford University

Stanford, CA 94305

finn@gauss.stanford.edu

Vaughan F. R. Jones

University of California

Berkeley, CA 94720

vfr@math.berkeley.edu

Steven Kerckhoff

Stanford University

Stanford, CA 94305

spk@gauss.stanford.edu
Martin Scharlemann

University of California

Santa Barbara, CA 93106

mgscharl@math.ucsb.edu

Gang Tian

Courant Institute

New York University

New York, NY 10012-1100

tiang@taotao.cims.nyu.edu

V. S. Varadarajan

University of California

Los Angeles, CA 90095-1555

vsv@math.ucla.edu

\section{SUPPORTING INSTITUTIONS}

\section{CALIFORNIA INSTITUTE OF TECHNOLOGY \\ NEW MEXICO STATE UNIVERSITY \\ OREGON STATE UNIVERSITY \\ STANFORD UNIVERSITY \\ UNIVERSITY OF ARIZONA \\ UNIVERSITY OF BRITISH COLUMBIA \\ UNIVERSITY OF CALIFORNIA \\ UNIVERSITY OF HAWAII}

\author{
UNIVERSITY OF MONTANA \\ UNIVERSITY OF NEVADA, RENO \\ UNIVERSITY OF OREGON \\ UNIVERSITY OF SOUTHERN CALIFORNIA \\ UNIVERSITY OF UTAH \\ UNIVERSITY OF WASHINGTON \\ WASHINGTON STATE UNIVERSITY
}

The supporting Institutions listed above contribute to the cost of publication of this Journal, but they are not owners or publishers and have no responsibility for its contents or policies.

Manuscripts must be prepared in accordance with the instructions provided on the inside back cover.

The Pacific Journal of Mathematics (ISSN 0030-8730) is published monthly except for July and August. Regular subscription rate: $\$ 215.00$ a year (10 issues). Special rate: $\$ 108.00$ a year to individual members of supporting institutions.

Subscriptions, orders for back issues published within the last three years, and changes of subscribers address should be sent to Pacific Journal of Mathematics, P.O. Box 4163, Berkeley, CA 94704-0163, U.S.A. Prior back issues are obtainable from Kraus Periodicals Co., Route 100, Millwood, NY 10546.

The Pacific Journal of Mathematics at the University of California, c/o Department of Mathematics, 981 Evans Hall, Berkeley, CA 94720 (ISSN 0030-8730) is published monthly except for July and August. Second-class postage paid at Berkeley, CA 94704, and additional mailing offices. POSTMASTER: send address changes to Pacific Journal of Mathematics, P.O. Box 6143, Berkeley, CA 94704-0163.

\author{
PUBLISHED BY PACIFIC JOURNAL OF MATHEMATICS at University of California, \\ Berkeley, CA 94720, A NON-PROFIT CORPORATION \\ This publication was typeset using AMS-LATEX, \\ the American Mathematical Society's TEX macro system. \\ Copyright (C) 1995 by Pacific Journal of Mathematics
}




\section{PACIFIC JOURNAL OF MATHEMATICS}

Volume $170 \quad$ No. $1 \quad$ September 1995

Generalized generalized spin models (four-weight spin models)

EIICHI BANNAI and ETSUKO BANNAI

Fine structure of the Mackey machine for actions of abelian groups with constant Mackey obstruction

SIEGFRIED ECHTERHOFF and JONATHAN ROSENBERG

The corestriction of valued division algebras over Henselian fields. I

YOON SUNG HWANG

The corestriction of valued division algebras over Henselian fields. II

YOON SUNG HWANG

The cohomology of expansive $\mathbb{Z}^{d}$-actions by automorphisms of compact, 105 abelian groups

ANATOLE KATOK and KLAUS SCHMIDT

The Anosov theorem for exponential solvmanifolds

EDWARD KEPPELMANN and CHRISTOPHER K. MCCORD

Projections of measures on nilpotent orbits and asymptotic multiplicities 161 of $K$-types in rings of regular functions. I

DONALD RAYMOND KING

On almost-everywhere convergence of inverse spherical transforms

Christopher Meaney and Elena Prestini

Characters of supercuspidal representations of $S L(n)$

Fiona AnNe Murnaghan

The cohomology of higher-dimensional shifts of finite type

KLAUS SCHMIDT

On Gorenstein surface singularities with fundamental genus $p_{f} \geqq 2$ which satisfy some minimality conditions

TADASHI TOMARU 